\title{
Universal Bounds for Large Determinants from Non-Commutative Hölder Inequalities in Fermionic Constructive Quantum Field Theory
}

\author{
J.-B. Bru W. de Siqueira Pedra
}

January 29, 2016

\begin{abstract}
Efficiently bounding large determinants is an essential step in non-relativistic fermionic constructive quantum field theory, because, together with the summability of the interaction and the covariance, it implies the absolute convergence of the perturbation expansion of all correlation functions in terms of powers of the strength $u \in \mathbb{R}$ of the interparticle interaction. We provide, for large determinants of fermionic convariances, sharp bounds which hold for all (bounded and unbounded, the latter not being limited to semibounded) one-particle Hamiltonians. We find the smallest universal determinant bound to be exactly 1 . In particular, the convergence of perturbation series at $u=0$ of any fermionic quantum field theory is ensured by the decay properties of the covariance and the interparticle interaction alone. Our proofs use Hölder inequalities for general non-commutative $L^{p}$-spaces derived by Araki and Masuda [AM].
\end{abstract}

\section{Contents}

1 Setup of the Problem and Main Results 2

1.1 Spaces of Antiperiodic Functions on Discrete Tori . . . . . . . . . 3

1.2 Discrete Time Covariance . . . . . . . . . . . . . . . 4

1.3 CAR $C^{*}$-Algebras Associated with Tree-Expansions . . . . . . . 5

1.4 Determinant Bounds in Constructive Quantum Field Theory . . . 7

1.5 Main Results . . . . . . . . . . . . . . . . . . . . . . . 10

1.6 Finite Dimensional Case and Hölder Inequalities for Schatten Norms 14 
2 Technical Proofs 18

2.1 Quasi-Free States . . . . . . . . . . . . . . . . . . . 18

2.2 Representation of Discrete Time Covariance by Quasi-Free States 24

2.3 Correlation Functions and Tomita-Takesaki Modular Theory . . . 30

\section{Setup of the Problem and Main Results}

The convergence of perturbation expansions in non-relativistic fermionic constructive quantum field theory at weak coupling is ensured if the interaction and the covariance are summable and if certain determinants arising in the expansion can be bounded efficiently. For any one-particle Hamiltonian we show here how to get such bounds on determinants from non-commutative Hölder inequalities. To our knowledge, such estimates are unknown for the unbounded case, even for semibounded (one-particle) Hamiltonians. The unbounded case is important, for instance, in the context of fermionic theories in the continuum.

The bounds on determinants (of fermionic covariances) obtained in this way turn out to be universal and sharp, in a sense to be made precise below (cf. (13) and Corollary 1.5). A consequence of these estimates is that the convergence of perturbation expansions in non-relativistic fermionic quantum field theory is implied by the summability properties of interaction and covariance alone. Similar to [dSPS], we give bounds which do not impose cutoffs on the Matsubara frequency, but the results obtained here are stronger than those of [dSPS] on determinants of fermionic covariances.

The paper is organized as follows: Definitions and notation are fixed in Sections 1.1-1.3. The problem of bounding large determinants and the importance of our results in the context of constructive quantum field theory are discussed in Section 1.4. Our main results are Theorem 1.3 and Corollaries 1.4-1.5 of Section 1.5. Our approach uses Hölder inequalities for general non-commutative $L^{p}$-spaces. See, e.g., [AM]. Section 1.6 illustrates the key arguments in the finite dimensional case via Hölder inequalities for Schatten norms. Detailed proofs are postponed to Section 2.

\section{Notation 1.1}

A norm on a generic vector space $\mathcal{Y}$ is denoted by $\|\cdot\|_{\mathcal{Y}}$ and the identity map of $\mathcal{Y}$ by $\mathbf{1}_{\mathcal{Y}}$. The space of all bounded linear operators on $\left(\mathcal{Y},\|\cdot\|_{\mathcal{Y}}\right)$ is denoted by $\mathcal{B}(\mathcal{Y})$. If $\mathcal{Y}$ is a Hilbert space, then $\langle\cdot, \cdot\rangle_{\mathcal{Y}}$ denotes its scalar product. Units of $C^{*}$-algebras are always denoted by 1 . 


\subsection{Spaces of Antiperiodic Functions on Discrete Tori}

We start by defining spaces of antiperiodic functions taking values in a fixed Hilbert space and next give the definition of the antiperiodic discrete delta function:

(i): Fix $\beta \in \mathbb{R}^{+}$, an even integer $n \in 2 \mathbb{N}$ and let

$$
\mathbb{T}_{n} \doteq\left\{-\beta+k n^{-1} \beta: k \in\{1,2, \ldots, 2 n\}\right\} \subset(-\beta, \beta]
$$

be the discrete torus of length $2 \beta$. This means that $-\beta \equiv \beta$. Pick any Hilbert space $\mathfrak{h}$ and let $\ell_{\text {ap }}^{2}\left(\mathbb{T}_{n} ; \mathfrak{h}\right)$ be the Hilbert space of functions from $\mathbb{T}_{n}$ to $\mathfrak{h}$ which are antiperiodic. That is here, for any $f \in \ell_{\mathrm{ap}}^{2}\left(\mathbb{T}_{n} ; \mathfrak{h}\right)$,

$$
f(\alpha+\beta)=-f(\alpha), \quad \alpha \in \mathbb{T}_{n} .
$$

The scalar product on $\ell_{\text {ap }}^{2}\left(\mathbb{T}_{n} ; \mathfrak{h}\right)$ is then defined to be

$$
\left\langle f_{1}, f_{2}\right\rangle_{\ell_{\mathrm{ap}}^{2}\left(\mathbb{T}_{n} ; \mathfrak{h}\right)} \doteq n^{-1} \beta \sum_{\alpha \in \mathbb{T}_{n}}\left\langle f_{1}(\alpha), f_{2}(\alpha)\right\rangle_{\mathfrak{h}}, \quad f_{1}, f_{2} \in \ell_{\mathrm{ap}}^{2}\left(\mathbb{T}_{n} ; \mathfrak{h}\right)
$$

The parameter $\beta$ is interpreted as being the inverse temperature in (fermionic and non-relativistic) quantum field theory, while $\mathfrak{h}$ refers to the so-called oneparticle Hilbert space in the same context. The use of antiperiodic functions on the torus is related to the KMS property of equilibrium states and the canonical anticommutation relations (CAR). The discretization of the torus, leading to $\mathbb{T}_{n}$ for $n \in 2 \mathbb{N}$, arises from the use of the Trotter-Kato formula in the construction of correlation functions of such KMS states as Berezin-Grassmann integrals.

(ii): We see the Hilbert space $\mathfrak{h}$ as a subset of $\ell_{\text {ap }}^{2}\left(\mathbb{T}_{n} ; \mathfrak{h}\right)$ by using the discrete delta function $\delta_{\text {ap }} \in \ell_{\text {ap }}^{2}\left(\mathbb{T}_{n} ; \mathbb{C}\right)$ defined by

$$
\delta_{\text {ap }}(\alpha) \doteq\left\{\begin{array}{lll}
0 & \text { if } & \alpha \notin\{0, \beta\} . \\
\frac{\beta^{-1} n}{2} & \text { if } & \alpha=0 . \\
-\frac{\beta^{-1} n}{2} & \text { if } & \alpha=\beta .
\end{array}\right.
$$

Vectors $\varphi$ of $\mathfrak{h}$ are viewed as antiperiodic functions $\hat{\varphi}$ of $\ell_{\text {ap }}^{2}\left(\mathbb{T}_{n} ; \mathfrak{h}\right)$ via the definition

$$
\hat{\varphi}(\alpha) \doteq \delta_{\text {ap }}(\alpha) \varphi, \quad \alpha \in \mathbb{T}_{n}
$$


Note that this identification is isometric up to a constant, since

$$
\left\langle\hat{\varphi}_{1}, \hat{\varphi}_{2}\right\rangle_{\ell_{\mathrm{ap}}^{2}\left(\mathbb{T}_{n} ; \mathfrak{h}\right)}=\frac{\beta^{-1} n}{2}\left\langle\varphi_{1}, \varphi_{2}\right\rangle_{\mathfrak{h}}, \quad \varphi_{1}, \varphi_{2} \in \mathfrak{h} .
$$

The discrete delta function $\delta_{\text {ap }}$ is useful here because of the property

$$
g * \delta_{\text {ap }}=g, \quad g \in \ell_{\text {ap }}^{2}\left(\mathbb{T}_{n} ; \mathfrak{h}\right),
$$

with the convolution being defined by

$$
g * f(\alpha) \doteq n^{-1} \beta \sum_{\vartheta \in \mathbb{T}_{n}} g(\alpha-\vartheta) f(\vartheta), g \in \ell_{\mathrm{ap}}^{2}\left(\mathbb{T}_{n} ; \mathfrak{h}\right), f \in \ell_{\mathrm{ap}}^{2}\left(\mathbb{T}_{n} ; \mathbb{C}\right), \alpha \in \mathbb{T}_{n}
$$

Indeed, $\delta_{\mathrm{ap}}$ is used below to construct the inverse of some discrete difference operator, see Equation (21).

\subsection{Discrete Time Covariance}

The discrete time covariance is an operator defined from (i) a self-adjoint operator acting on the Hilbert space $\mathfrak{h}$ and (ii) the discrete derivative operator acting on the space $\ell_{\text {ap }}^{2}\left(\mathbb{T}_{n} ; \mathfrak{h}\right)$ of antiperiodic functions:

(i): Any (possibly unbounded) operator $A$ acting on $\mathfrak{h}$ with domain $\operatorname{dom}(A)$ is viewed as an operator $\hat{A}$ with domain

$$
\ell_{\text {ap }}^{2}\left(\mathbb{T}_{n} ; \operatorname{dom}(\hat{A})\right) \subset \ell_{\text {ap }}^{2}\left(\mathbb{T}_{n} ; \mathfrak{h}\right)
$$

by the definition

$$
[\hat{A} f](\alpha) \doteq A(f(\alpha)), \quad f \in \ell_{\mathrm{ap}}^{2}\left(\mathbb{T}_{n} ; \operatorname{dom}(\hat{A})\right), \alpha \in \mathbb{T}_{n} .
$$

If $A=H=H^{*}$ then $\hat{H}$ is also self-adjoint on the Hilbert space $\ell_{\text {ap }}^{2}\left(\mathbb{T}_{n} ; \mathfrak{h}\right)$ of antiperiodic functions.

The (possibly unbounded) self-adjoint operator $H=H^{*}$ acting on the Hilbert space $\mathfrak{h}$ is viewed as the so-called one-particle Hamiltonian in (fermionic and non-relativistic) quantum field theory. Indeed, its second quantization refers to the free part of the full interaction of the fermion system.

(ii): The discrete derivative operator $\partial \in \mathcal{B}\left(\ell_{\text {ap }}^{2}\left(\mathbb{T}_{n} ; \mathfrak{h}\right)\right)$ is the bounded operator defined by

$$
\partial f(\alpha) \doteq \beta^{-1} n\left(f\left(\alpha+n^{-1} \beta\right)-f(\alpha)\right), \quad f \in \ell_{\mathrm{ap}}^{2}\left(\mathbb{T}_{n} ; \mathfrak{h}\right), \alpha \in \mathbb{T}_{n} .
$$


It is a normal invertible operator. Combining (7) and (8) we remark that

$$
[\hat{A}, \partial] \doteq \hat{A} \partial-\partial \hat{A}=0
$$

for any operator $A$ acting on $\mathfrak{h}$. Therefore, since

$$
\inf \operatorname{spec}(|\operatorname{Im} \partial|)>0,
$$

if $H=H^{*}$ is any self-adjoint operator acting on $\mathfrak{h}$, then $(\partial+\hat{H})$ is a (possibly unbounded) normal operator with bounded inverse. The discrete time covariance is thus defined to be

$$
C_{H} \doteq-2(\partial+\hat{H})^{-1} \in \mathcal{B}\left(\ell_{\mathrm{ap}}^{2}\left(\mathbb{T}_{n} ; \mathfrak{h}\right)\right) .
$$

This type of operator appears as the covariance of Gaussian Berezin-Grassmann integrals used in the construction of correlation functions for systems of interacting fermions, see [S]. The discrete time derivative is related to the corresponding Trotter-Kato product formula used to define such integrals, as already mentioned in Section 1.1.

\subsection{CAR $C^{*}$-Algebras Associated with Tree-Expansions}

We now define CAR $C^{*}$-algebras $\operatorname{CAR}(\mathfrak{h} \otimes \mathbb{M})$ constructed from a fixed $\mathfrak{h}$ and some finite-dimensional Hilbert spaces $\mathbb{M}$, having in mind Brydges-Kennedy tree expansions:

(i): Fix $m \in \mathbb{N}$. Let $\mathfrak{M} \in \operatorname{Mat}(m, \mathbb{R})$ be a real $m \times m$ matrix that is positive, i.e., $\overline{\mathfrak{M}} \geq 0$. Recall that the positivity of $\mathfrak{M}$ includes its symmetry. Finally, to avoid triviality of assertions, we assume $\mathfrak{M} \neq 0$.

Here, we have in mind positive matrices appearing in the so-called BrydgesKennedy tree expansions which have the following structure: For each non-oriented graph $\mathfrak{g}$ with $m$ vertices, all functions $\boldsymbol{\alpha} \in[0,1]^{\mathfrak{g}}$ and any parameter $s \in[0,1]$, we define the subgraph

$$
\mathfrak{g}(\boldsymbol{\alpha}, s) \doteq \mathfrak{g} \backslash\{\ell \in \mathfrak{g}: \boldsymbol{\alpha}(\ell) \geq s\} \subset \mathfrak{g}
$$

In fact, only minimally connected graphs (trees) $\mathfrak{g}$ are relevant for the BrydgesKennedy tree expansions. Let $\mathcal{R}_{\mathfrak{g}(\boldsymbol{\alpha}, s)} \subset\{1, \ldots, m\}^{2}$ denote the smallest equivalence relation for which one has $(k, l) \in \mathcal{R}_{\mathfrak{g}(\boldsymbol{\alpha}, s)}$ for all $k, l \in\{1, \ldots, m\}$ 
such that the line $\{k, l\}$ belongs to the graph $\mathfrak{g}(\boldsymbol{\alpha}, s)$. Then, for any $t \in[0,1]$, $\mathfrak{M}=\mathfrak{M}(\mathfrak{g}, \boldsymbol{\alpha}, t)$ is the symmetric positive $m \times m$ real matrix defined by

$$
[\mathfrak{M}(\mathfrak{g}, \boldsymbol{\alpha}, t)]_{k, l} \doteq \int_{0}^{t} \mathbf{1}\left[(k, l) \in \mathcal{R}_{\mathfrak{g}(\boldsymbol{\alpha}, s)}\right] \mathrm{d} s, \quad k, l \in\{1, \ldots, m\}
$$

See for instance [AR, BK, SW].

(ii): The (generic) non-vanishing positive matrix $\mathfrak{M}$ gives rise to a positive sesquilinear form defined on $\mathbb{C}^{m}$ by

$$
\left\langle\left(x_{1}, \ldots, x_{m}\right),\left(y_{1}, \ldots, y_{m}\right)\right\rangle_{\mathbb{C}^{m}}^{\mathfrak{M}} \doteq \sum_{p, q=1}^{m} \overline{x_{p}} y_{q} \mathfrak{M}_{p, q} .
$$

In general, this sesquilinear form is degenerated. The vector space $\mathbb{M}$ is then defined to be the quotient

$$
\mathbb{M} \doteq \mathbb{C}^{m} /\left\{x \in \mathbb{C}^{m}:\langle x, x\rangle_{\mathbb{C}^{m}}^{\mathfrak{M}}=0\right\}
$$

Then, as usual, we introduce a scalar product on $\mathbb{M}$ as

$$
\langle[x],[y]\rangle_{\mathbb{M}} \doteq\langle x, y\rangle_{\mathbb{C}^{m}}^{\mathfrak{M}}, \quad x, y \in \mathbb{C}^{m},
$$

and $\mathbb{M}$ denotes the Hilbert space $\left(\mathbb{M},\langle\cdot, \cdot\rangle_{\mathbb{M}}\right)$. Using the notation $\mathfrak{e}_{k} \doteq\left[e_{k}\right] \in \mathbb{M}$, where $\left\{e_{k}\right\}_{k=1}^{m}$ is the canonical basis of $\mathbb{C}^{m}$, note that

$$
\mathfrak{M}_{k, l}=\left\langle\mathfrak{e}_{k}, \mathfrak{e}_{l}\right\rangle_{\mathbb{M}}, \quad k, l \in\{1, \ldots, m\} .
$$

(iii): The (extended) CAR $C^{*}$-algebra associated with $\mathfrak{M}$ is the unital $C^{*}$-algebra $\overline{\mathrm{CAR}}(\mathfrak{h} \otimes \mathbb{M})$ generated by the unit 1 and the family $\{a(\Psi)\}_{\Psi \in \mathfrak{h} \otimes \mathbb{M}}$ of elements satisfying the canonical anticommutation relations (CAR), see (33)-(34) with $\mathcal{H}=\mathfrak{h} \otimes \mathbb{M}$. Notice that such a family always exists and two families satisfying these CAR are related to each other by a unique $*$-automorphism on the $C^{*}$-algebra $\mathrm{CAR}(\mathfrak{h} \otimes \mathbb{M})$. See, e.g., [BR2, Theorem 5.2.5].

The operator $a(\Psi) \in \mathrm{CAR}(\mathfrak{h} \otimes \mathbb{M})$ is the annihilation operator associated with $\Psi \in \mathfrak{h} \otimes \mathbb{M}$ whereas its adjoint

$$
a^{+}(\Psi) \doteq a(\Psi)^{*}, \quad \Psi \in \mathfrak{h} \otimes \mathbb{M},
$$

is the corresponding creation operator in fermionic quantum field theory. 
Considering that $\mathfrak{h}$ represents the one-particle Hilbert space, $\operatorname{CAR}(\mathfrak{h})$ is the $C^{*}$-algebra that allows to represent the corresponding many-fermion system within the algebraic formulation of quantum mechanics. The extension of this $C^{*}-$ algebra to $\operatorname{CAR}(\mathfrak{h} \otimes \mathbb{M})$ is pivotal. Indeed, the construction of correlation functions for (interacting) fermion systems via Brydges-Kennedy tree expansions imposes to control determinants of matrices with entries depending on $\mathfrak{M}=\mathfrak{M}(\mathfrak{g}, \boldsymbol{\alpha}, t)$, $\mathfrak{g}$ being an arbitrary tree. See (12). Such determinants are naturally expressed through limits of quasi-free states on the $C^{*}$-algebra $\mathrm{CAR}(\mathfrak{h} \otimes \mathbb{M})$. See Theorem 1.3 as well as Section 2.2.

\subsection{Determinant Bounds in Constructive Quantum Field The- ory}

Correlation functions of interacting fermions can be constructed by perturbation series in the regime of weak couplings. In this context, the self-adjoint (possibly unbounded) operator $H=H^{*}$ acting on $\mathfrak{h}$ is the generator of the unperturbed dynamics of the fermion system.

Now, suppose, for simplicity, that $\mathfrak{h}$ is a separable Hilbert space with ONB $\left\{\varphi_{i}\right\}_{i \in \mathbb{I}}, \mathbb{I}$ being countable, and set

$$
\boldsymbol{\omega}_{H, \kappa} \doteq \limsup _{n \rightarrow \infty} \sup _{\mathfrak{i} \in \mathbb{I}}\left\{n^{-1} \beta \sum_{\vartheta \in \mathbb{T}_{n}} \sum_{q \in \mathbb{I}}\left|\left\langle\varphi_{q},\left(C_{H} \kappa(\hat{H}) \hat{\varphi}_{\mathfrak{i}}\right)(\vartheta)\right\rangle_{\mathfrak{h}}\right|\right\}
$$

for any $\beta \in \mathbb{R}^{+}, H=H^{*}$ and measurable function $\kappa$ from $\mathbb{R}$ to $\mathbb{R}_{0}^{+}$. See (3), (7) and (9). We have in mind cutoff functions $\kappa: \mathbb{R} \rightarrow[0,1]$.

Another essential quantity in non-relativistic fermionic constructive quantum field theory is the so-called determinant bound of $H$ and $\kappa$ defined as follows:

\section{Definition 1.2 (Determinant bounds)}

The parameter $\gamma_{H, \kappa} \in \mathbb{R}^{+}$is a determinant bound of $H=H^{*}$ and the measurable function $\kappa: \mathbb{R} \rightarrow \mathbb{R}_{0}^{+}$if, for any $\beta \in \mathbb{R}^{+}, n \in 2 \mathbb{N}, m, N \in \mathbb{N}, \mathfrak{M} \in \operatorname{Mat}(m, \mathbb{R})$ with $\mathfrak{M} \geq 0$, and all parameters

$$
\left\{\left(\alpha_{q}, \mathfrak{i}_{q}, j_{q}\right)\right\}_{q=1}^{2 N} \subset \mathbb{T}_{n} \cap[0, \beta) \times \mathbb{I} \times\{1, \ldots, m\},
$$

the following bound holds true:

$$
\left|\operatorname{det}\left[\mathfrak{M}_{j_{k}, j_{N+l}}\left\langle\varphi_{\mathfrak{i}_{N+l}},\left(C_{H} \kappa(\hat{H}) \hat{\varphi}_{\mathfrak{i}_{k}}\right)\left(\alpha_{k}-\alpha_{N+l}\right)\right\rangle_{\mathfrak{h}}\right]_{k, l=1}^{N}\right| \leq \gamma_{H, \kappa}^{2 N} \prod_{q=1}^{2 N} \mathfrak{M}_{j_{q}, j_{q}}^{1 / 2} \text {. }
$$


Indeed, let $u \in \mathbb{R}$ be the coupling constant of the considered interacting fermion system, which means that $u$ quantifies the strength of the interparticle interaction. Then, it can be shown that, if the parameter $\boldsymbol{\omega}_{H, \mathbf{1}_{\mathbb{R}}} \gamma_{H, \mathbf{1}_{\mathbb{R}}}^{2}|u|$ is small enough, then the perturbation expansion of all correlation functions in terms of powers of $u$ converges absolutely. More precisely, all correlation functions are analytic functions of the coupling $u$ at $u=0$ with analyticity radius of order $\boldsymbol{\omega}_{H, \mathbf{1}_{\mathbb{R}}}^{-1} \gamma_{H, \mathbf{1}_{\mathbb{R}}}^{-2}$. See for instance [AR, SW].

The use of the cutoff function $\kappa$ is important in multiscale analyses of correlation functions of interacting fermion systems. Indeed, even for couplings $|u|$ much larger than the convergence radius $\boldsymbol{\omega}_{H, \mathbf{1}_{\mathbb{R}}}^{-1} \gamma_{H, \mathbf{1}_{\mathbb{R}}}^{-2}$ correlations functions can still be constructed via multiscale schemes related to the Wilson renormalization group: Take a family $\left\{\kappa_{L}\right\}_{L \in \mathbb{N}}$ of measurable functions from $\mathbb{R}$ to $[0,1]$ such that

$$
\sum_{L=1}^{\infty} \kappa_{L}(x)=1, \quad x \in \mathbb{R} .
$$

(I.e., the family is a partition of unity.) If $\boldsymbol{\omega}_{H, \kappa_{L}} \gamma_{H, \kappa_{L}}^{2}|u|$ is small enough for all $L \in \mathbb{N}$, then, up to technical details, the perturbation series at scale $L$ in terms of powers of $u$ converges absolutely. In general, the smallness of the parameters $\boldsymbol{\omega}_{H, \kappa_{L}} \gamma_{H, \kappa_{L}}^{2}|u|$ at all scales is a much weaker condition than the smallness of $\boldsymbol{\omega}_{H, \mathbf{1}_{\mathbb{R}}} \gamma_{H, \mathbf{1}_{\mathbb{R}}}^{2}|u|$. See for instance [dSP].

Note that the form of cutoff function we consider does not depend on the $\alpha$ variables, that is, the dependency on the Matsubara frequency of covariance does not need to be regularized, in contrast to other approaches like for instance [GM, BGPS, GMP].

Indeed, coming back to the estimate of the form (12), one easily shows from the Gram bound for determinants that

$$
\begin{aligned}
& \operatorname{det}\left[\mathfrak { M } _ { j _ { k } , j _ { N + l } } \left\langle\varphi_{\mathfrak{i}_{N+l}},\right.\right.\left.\left.\left(C_{H} \kappa(\hat{H}) \hat{\varphi}_{\mathfrak{i}_{k}}\right)\left(\alpha_{k}-\alpha_{N+l}\right)\right\rangle_{\mathfrak{h}}\right]_{k, l=1}^{N} \mid \\
& \leq\left\|C_{H}\right\|_{\mathcal{B}\left(\ell_{\mathrm{ap}}^{2}\left(\mathbb{T}_{n} ; \mathfrak{h}\right)\right)}^{N} \prod_{q=1}^{2 N}\left\|\sqrt{\kappa(\hat{H}) \hat{\varphi}_{\mathfrak{i}_{q}}}\right\|_{\ell_{\mathrm{ap}}^{2}\left(\mathbb{T}_{n} ; \mathfrak{h}\right)} \mathfrak{M}_{j_{q}, j_{q}}^{1 / 2}
\end{aligned}
$$

This kind of estimate gives no finite determinant bound of $H$ and $\kappa$ because, in general, the norm of $C_{H}$ diverges, as $n \rightarrow \infty$. This problem appears already for bounded $H \in \mathcal{B}(\mathfrak{h})$ when $0 \in \operatorname{spec}(H)$, because in this case

$$
\left\|C_{H}\right\|_{\mathcal{B}\left(\ell_{\mathrm{ap}}^{2}\left(\mathbb{T}_{n} ; \mathfrak{h}\right)\right)}^{1 / 2}=\mathcal{O}(\sqrt{n}) \quad \text { and } \quad\left\|\hat{\varphi}_{\mathrm{i}_{q}}\right\|_{\ell_{\mathrm{ap}}^{2}\left(\mathbb{T}_{n} ; \mathfrak{h}\right)}=\mathcal{O}(\sqrt{n}),
$$


as $n \rightarrow \infty$. See (4). Nevertheless, similar to the multiscale analysis presented above, one can tackle this problem by using the Gram bound as previously for some regularized covariances $C_{H} \hat{\kappa}_{L}(\hat{H}, i \partial)$ at every $L \in \mathbb{N}$. Here, for any $L \in \mathbb{N}$, $\hat{\kappa}_{L}: \mathbb{R}^{2} \rightarrow[0,1]$ is some measurable function of two variables in such a way that

$$
\sum_{L=1}^{\infty} \hat{\kappa}_{L}(x, y)=\kappa(x), \quad x, y \in \mathbb{R} .
$$

This decomposition can be chosen such that there are constants $\hat{\gamma}_{L} \in \mathbb{R}^{+}, L \in \mathbb{N}$, which at least do not depend on $n \in 2 \mathbb{N}$ and meanwhile satisfy

$\left|\operatorname{det}\left[\mathfrak{M}_{j_{k}, j_{N+l}}\left\langle\varphi_{\mathfrak{i}_{N+l}},\left(C_{H} \hat{\kappa}_{L}(\hat{H}, i \partial) \hat{\varphi}_{\mathfrak{i}_{k}}\right)\left(\alpha_{k}-\alpha_{N+l}\right)\right\rangle_{\mathfrak{h}}\right]_{k, l=1}^{N}\right| \leq \hat{\gamma}_{L}^{2 N} \prod_{q=1}^{2 N} \mathfrak{M}_{j_{q}, j_{q}}^{1 / 2}$

As already mentioned, such a bound follows from the usual Gram bound for determinants. This kind of strategy is used for instance in [BGPS, Section 3], [GM, Section 3.2], (more recently) [GMP, Section 5.A.], and in many others works. [dSPS] shows that this multiscale analysis for the so-called the Matsubara UV problem is not necessary, by proving a new bound for determinants that generalizes the original Gram bound, see [dSPS, Theorem 1.3]. Note finally that using multiscale analysis to treat the Matsubara UV problem can, moreover, render useful properties of the full covariance less transparent. Hence, avoiding this kind of procedure brings various technical benefits.

In the same spirit, we derive direct bounds of the type (12) that do not need the UV regularization of the Matsubara frequency. One technical advantage of the approach we present here is that the given covariance does not need to be decomposed as in [dSPS, Eq. (8)] in order to obtain determinant bounds. Moreover, our estimates are sharp (or optimal) and hold true for all (possibly unbounded, the latter not being limited to semibounded) one-particle Hamiltonians. Observe that [dSPS] gives sharp estimates up to a prefactor 2 for the class of bounded operators it applies, see [dSPS, Theorem 2.4 and discussions below it].

In this paper we show the (possibly infinite) general bound

$$
\begin{aligned}
\mathfrak{x} & \doteq \sup \left\{\inf \left\{\gamma_{H, \mathbf{1}_{\mathbb{R}}} \in \mathbb{R}^{+}: \gamma_{H, \mathbf{1}_{\mathbb{R}}} \text { determinant bound of } H \text { and } \mathbf{1}_{\mathbb{R}}\right\}\right. \\
& \left.: \quad H=H^{*} \text { acting on a separable Hilbert space } \mathfrak{h} \text { with } \operatorname{ONB}\left\{\varphi_{\mathfrak{i}}\right\}_{\mathfrak{i} \in \mathbb{I}}\right\},
\end{aligned}
$$


named here the universal determinant bound, is equal to $\mathfrak{x}=1$. (Even if the class of all separable Hilbert spaces is not a set, the supremum is well-defined because of the separation axiom.) In particular, the convergence of perturbation series at $u=0$ of any non-relativistic fermionic quantum field theory (possibly in the continuum) is ensured by the smallness of the positive parameter $\boldsymbol{\omega}_{H, \mathbf{1}_{\mathbb{R}}}$, i.e., if the interaction and the covariance are summable, only. To our knowledge, such estimates are unknown for unbounded self-adjoint operators $H$, even for semibounded ones. Similar statements can also be derived while taking into account the (cutoff) function $\kappa$, see Corollary 1.4. Note that we only consider separable Hilbert spaces in (13) to avoid technical issues.

\subsection{Main Results}

To prove our estimates, we rewrite the determinant of (12) by using cyclic representations of quasi-free states on the $C^{*}$-algebra $\mathrm{CAR}(\mathfrak{h} \otimes \mathbb{M})$. This allows us to use the bound [AM, (A.2)], which can be viewed as Hölder inequalities for general non-commutative $L^{p}$-spaces. This yields the following assertions on determinants of fermionic covariances:

\section{Theorem 1.3 (Representation of determinants of fermionic covariances)}

Let $\mathfrak{h}$ be any separable Hilbert space. Take $\beta \in \mathbb{R}^{+}, m \in \mathbb{N}, n \in 2 \mathbb{N}$, any selfadjoint operator $H=H^{*}$ acting on $\mathfrak{h}$, and a non-vanishing $\mathfrak{M} \in \operatorname{Mat}(m, \mathbb{R})$ with $\mathfrak{M} \geq 0$. Then there are von Neumann algebras $\mathcal{X}_{\nu} \subset \mathcal{B}\left(\mathfrak{H}_{\nu}\right)$, cyclic and separating unit vectors $\eta_{\nu} \in \mathfrak{H}_{\nu}$ (for $\mathcal{X}_{\nu}$ ) and $C^{*}$-homomorphisms $\varkappa_{\nu}$ (from $\mathrm{CAR}(\mathfrak{h} \otimes \mathbb{M})$ to $\left.\mathcal{X}_{\nu}\right)$, where $\nu \in \mathbb{R}^{+}$, such that for each bounded measurable positive function $\kappa$ from $\mathbb{R}$ to $\mathbb{R}_{0}^{+}$, all parameters

$$
\left\{\left(\alpha_{q}, \varphi_{q}, j_{q}\right)\right\}_{q=1}^{2 N} \subset \mathbb{T}_{n} \cap[0, \beta) \times \mathfrak{h} \times\{1, \ldots, m\},
$$

and for any permutation $\pi$ of $2 N \in \mathbb{N}$ elements with sign $(-1)^{\pi}$ so that ${ }^{1}$

$\vartheta_{q} \doteq \beta^{-1}\left(\tilde{\alpha}_{\pi^{-1}(q)}-\tilde{\alpha}_{\pi^{-1}(q-1)}\right) \geq 0, \alpha_{\pi^{-1}(q)}-\alpha_{\pi^{-1}(q-1)} \geq 0, q \in\{2, \ldots, 2 N\}$,

where $\tilde{\alpha}_{q} \doteq \alpha_{q}$ for $q \in\{1, \ldots, N\}$ and $\tilde{\alpha}_{q} \doteq \alpha_{q}+n^{-1} \beta$ for $q \in\{N+1, \ldots, 2 N\}$,

\footnotetext{
${ }^{1}$ The conditions on $\pi$ impose that it is a permutation of $2 N$ elements which orders the numbers $\alpha_{q}, q \in\{1, \ldots, 2 N\}$, in the following way: $\pi(k)<\pi(l)$ whenever $\alpha_{k}<\alpha_{l}$ for $k, l \in\{1, \ldots, 2 N\}$ while $\pi(k)<\pi(N+l)$ whenever $\alpha_{k}=\alpha_{N+l}$ for $k, l \in\{1, \ldots, N\}$.
} 
the following assertion holds true:

$$
\begin{gathered}
\operatorname{det}\left[\mathfrak{M}_{j_{k}, j_{N+l}}\left\langle\varphi_{N+l},\left(C_{H} \kappa(\hat{H}) \hat{\varphi}_{k}\right)\left(\alpha_{k}-\alpha_{N+l}\right)\right\rangle_{\mathfrak{h}}\right]_{k, l=1}^{N} \\
=(-1)^{\pi} \lim _{\nu \rightarrow \infty}\left\langle\Delta_{\nu}^{\frac{1}{2}-\beta^{-1} \tilde{\alpha}_{\pi^{-1}(p-1)} x_{p-1}^{*} \Delta_{\nu}^{\vartheta_{p-1}} \cdots x_{2}^{*} \Delta_{\nu}^{\vartheta_{2}} x_{1}^{*} \eta_{\nu},}\right. \\
\left.\Delta_{\nu}^{\beta^{-1} \tilde{\alpha}_{\pi^{-1}(p)}-\frac{1}{2}} x_{p} \Delta_{\nu}^{\vartheta_{p+1}} x_{p+1} \cdots \Delta_{\nu}^{\vartheta_{N}} x_{N} \eta_{\nu}\right\rangle_{\mathfrak{H}_{\nu}} .
\end{gathered}
$$

The integer $p$ is defined to be the smallest element of $\{1, \ldots, 2 N\}$ so that $\tilde{\alpha}_{\pi(p)} \geq$ $\beta / 2 . \Delta_{\nu}$ is the modular operator associated with the pair $\left(\mathcal{X}_{\nu}, \eta_{\nu}\right)$. For $q \in$ $\{1, \ldots, 2 N\}$ such that $\pi^{-1}(q) \in\{1, \ldots, N\}$,

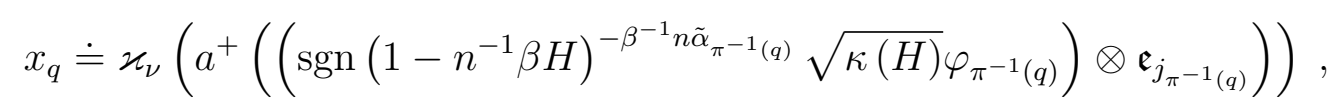

while for $q \in\{1, \ldots, 2 N\}$ such that $\pi^{-1}(q) \in\{N+1, \ldots, 2 N\}$,

$$
x_{q} \doteq \varkappa_{\nu}\left(a\left(\left(\operatorname{sgn}\left(1-n^{-1} \beta H\right)^{\beta^{-1} n \tilde{\alpha}_{\pi^{-1}(q)}} \sqrt{\kappa(H)} \varphi_{\pi^{-1}(q)}\right) \otimes \mathfrak{e}_{j_{\pi^{-1}}(q)}\right)\right) .
$$

Here, $\operatorname{sgn}$ is the sign function defined as follows: $\operatorname{sgn}(x) \doteq 1$ for $x \in \mathbb{R}_{0}^{+}$and $\operatorname{sgn}(x) \doteq-1$ otherwise.

Proof: $\quad$ First of all, we assume the Hilbert space $\mathfrak{h}$ to be separable to avoid technical issues. Recall also that $\mathfrak{M} \neq 0$ to avoid triviality of assertions.

Combining Lemma 2.2 and Corollary 2.4 with the construction done in Section 2.3, in particular Equation (74), one gets the assertion when all functions $\varphi_{1}, \ldots, \varphi_{N} \in \mathfrak{D} \subset \mathfrak{h}$ belong the dense space (57). To extend it to all $\varphi_{1}, \ldots, \varphi_{N} \in$ $\mathfrak{h}$, by (73), note that both sides of Equation (14) are continuous with respect to $\varphi_{1}, \ldots, \varphi_{N}$.

The heuristics behind this proof is as follows: We write the determinant of Equation (14) as the limit $\nu \rightarrow \infty$ of correlation functions associated with faithful quasi-free states from which we construct the von Neumann algebras $\mathcal{X}_{\nu} \subset$ $\mathcal{B}\left(\mathfrak{H}_{\nu}\right)$, the cyclic and separating unit vectors $\eta_{\nu} \in \mathfrak{H}_{\nu}$ and the $C^{*}$-homomorphisms $\varkappa_{\nu}$. See Section 2.3.

This construction is based on the following observations: Fix any $n \in 2 \mathbb{N}$. For any $\nu \in \mathbb{R}^{+}$, define the function

$$
\digamma_{\nu}(\lambda) \doteq\left\{\begin{array}{lll}
-\beta^{-1} n \ln \left|1-n^{-1} \beta \lambda\right| & \text { if } & \lambda \in \mathbb{R} \backslash\left\{\beta^{-1} n\right\} . \\
\nu & \text { if } & \lambda=\beta^{-1} n .
\end{array}\right.
$$


The relevant quasi-free state on $\operatorname{CAR}(\mathfrak{h} \otimes \mathbb{M})$ here is the KMS state at inverse temperature $\beta \in \mathbb{R}^{+}$associated with the one-particle dynamics $\left\{\mathrm{e}^{i t H_{\nu}}\right\}_{t \in \mathbb{R}}$, where

$$
H_{\nu} \doteq \digamma_{\nu}(H), \quad \nu \in \mathbb{R}^{+} .
$$

At fixed $\lambda \in \mathbb{R}$ and large $n \gg 1$, note that

$$
\mathrm{e}^{\mp \beta \digamma_{\nu}(\lambda)}=\left(1-n^{-1} \beta \lambda\right)^{ \pm n}=\mathrm{e}^{\mp \beta \lambda}+o(1)
$$

is a well-known approximation of the exponential function $\mathrm{e}^{\mp \beta \lambda}$. In particular, $H_{\nu}$ can be viewed as an approximation of $H$.

More precisely, this KMS state is the faithful quasi-free state on the $C^{*}$ algebra $\mathrm{CAR}(\mathfrak{h} \otimes \mathbb{M})$ with symbol

$$
S_{\nu} \doteq \frac{1}{1+\mathrm{e}^{\beta H_{\nu} \otimes \mathbf{1}_{\mathbb{M}}}}=\frac{1}{1+\mathrm{e}^{\beta H_{\nu}}} \otimes \mathbf{1}_{\mathbb{M}} \in \mathcal{B}(\mathfrak{h} \otimes \mathbb{M})
$$

See Section 2.1, observing that $0<S_{\nu} \leq \mathbf{1}_{\mathfrak{h} \otimes \mathbb{M}}$.

The relevance of the function $\digamma_{\nu}$ results from the following facts: Let $\lambda \in \mathbb{R}$. The parameter $\lambda$ plays the role of the self-adjoint operator $H$, by the spectral theorem. The antiperiodic function $g_{\lambda}^{(\infty)}: \mathbb{R} \rightarrow \mathbb{R}$ defined by

$$
g_{\lambda}^{(\infty)}(\alpha) \doteq \frac{\mathrm{e}^{-\alpha \lambda}}{1+\mathrm{e}^{\beta \lambda}}, \quad \alpha \in(-\beta, 0]
$$

solves the differential equation

$$
-y^{\prime}-\lambda y=\sum_{l=-\infty}^{\infty}(-1)^{l} \delta_{\beta l} .
$$

Here, $\delta_{x}$ is the delta distribution at $x \in \mathbb{R}$. In the discrete case, i.e., on the discrete torus $\mathbb{T}_{n} \subset[-\beta, \beta]$, similar to (19), one computes that the antiperiodic function $g_{\lambda} \in \ell_{\mathrm{ap}}^{2}\left(\mathbb{T}_{n} ; \mathbb{C}\right)$ defined by

$$
g_{\lambda}(\alpha) \doteq \frac{\left(1-n^{-1} \beta \lambda\right)^{\beta^{-1} n\left(\alpha-n^{-1} \beta\right)}}{1+\mathrm{e}^{\beta \digamma_{\nu}(\lambda)}}, \quad \alpha \in \mathbb{T}_{n} \cap(-\beta, 0],
$$

for $\lambda \neq \beta^{-1} n$, is the unique solution on $\ell_{\mathrm{ap}}^{2}\left(\mathbb{T}_{n} ; \mathbb{C}\right)$ of the difference equation

$$
-\beta^{-1} n\left(f\left(\alpha+n^{-1} \beta\right)-f(\alpha)\right)-\lambda f(\alpha)=2 \delta_{\text {ap }}(\alpha), \quad \alpha \in \mathbb{T}_{n},
$$


with the discrete delta function $\delta_{\mathrm{ap}} \in \ell_{\mathrm{ap}}^{2}\left(\mathbb{T}_{n} ; \mathbb{C}\right)$ being defined by Equation (2). Compare also (21) with (8) and (9). Note that we take $n \in 2 \mathbb{N}$ to ensure that

$$
\left(1-n^{-1} \beta \lambda\right)^{n}=\left|1-n^{-1} \beta \lambda\right|^{n}=\mathrm{e}^{-\beta \digamma_{\nu}(\lambda)}
$$

and observe meanwhile that $\alpha \beta^{-1} n \in \mathbb{Z}$ if $\alpha \in \mathbb{T}_{n}$. Therefore, for any $\lambda \neq \beta^{-1} n$, $g_{\lambda}(\alpha)=\left(\operatorname{sgn}\left(1-n^{-1} \beta \lambda\right)\right)^{\beta^{-1} n\left(n^{-1} \beta-\alpha\right)} \frac{\mathrm{e}^{-\left(\alpha-n^{-1} \beta\right) \digamma_{\nu}(\lambda)}}{1+\mathrm{e}^{\beta \digamma_{\nu}(\lambda)}}, \quad \alpha \in \mathbb{T}_{n} \cap(-\beta, 0]$.

Recall that $\operatorname{sgn}$ is the sign function defined here as follows: $\operatorname{sgn}(x) \doteq 1$ for $x \in \mathbb{R}_{0}^{+}$and $\operatorname{sgn}(x) \doteq-1$ otherwise.

On the other hand, the (unique) solution on $\ell_{\mathrm{ap}}^{2}\left(\mathbb{T}_{n} ; \mathbb{C}\right)$ of the difference equation (21) for $\lambda=\beta^{-1} n$ is equal to

$$
g_{\beta^{-1} n}(\alpha) \doteq\left\{\begin{array}{lll}
0 & \text { if } & \alpha \in \mathbb{T}_{n} \backslash\left\{0,-\beta+n^{-1} \beta\right\} . \\
-1 & \text { if } & \alpha=n^{-1} \beta . \\
1 & \text { if } & \alpha=-\beta+n^{-1} \beta .
\end{array}\right.
$$

In particular, we can write this function as the following limit:

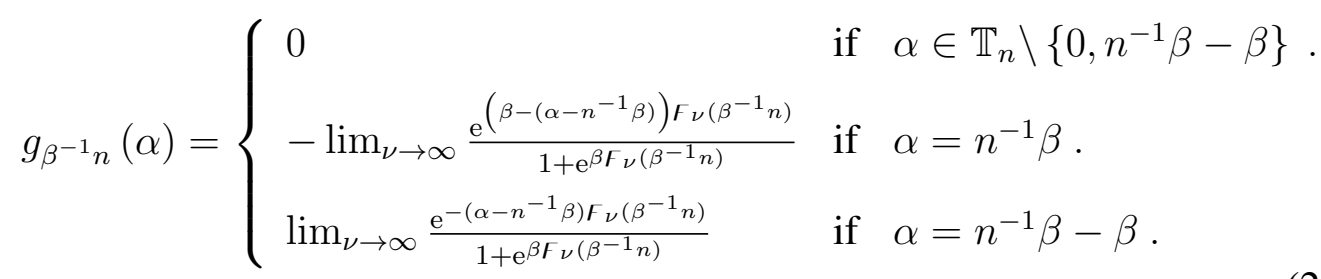

Compare (23) with (22). Up to the observation (17) and the special case $\lambda=$ $\beta^{-1} n$, the qualitative difference between (19) and (22) concerns the replacement of $\alpha$ in (19) by $\alpha-n^{-1} \beta$ in (22) and the prefactor

$$
\left(\operatorname{sgn}\left(1-n^{-1} \beta \lambda\right)\right)^{\beta^{-1} n\left(\alpha-n^{-1} \beta\right)},
$$

which give rise to the definitions of $\tilde{\alpha}_{q}$ and $x_{q}$, respectively.

\section{Corollary 1.4 (Determinant bounds)}

Under the assumptions of Theorem 1.3,

$$
\begin{array}{r}
\left|\operatorname{det}\left[\mathfrak{M}_{j_{k}, j_{N+l}}\left\langle\varphi_{N+l},\left(C_{H} \kappa(\hat{H}) \hat{\varphi}_{k}\right)\left(\alpha_{k}-\alpha_{N+l}\right)\right\rangle_{\mathfrak{h}}\right]_{k, l=1}^{N}\right| \\
\leq \prod_{q=1}^{2 N}\left\|\sqrt{\kappa(H)} \varphi_{q}\right\|_{\mathfrak{h}} \mathfrak{M}_{j_{q}, j_{q}}^{1 / 2}
\end{array}
$$


Compare with Definition 1.2.

Proof: $\quad$ This corollary is a direct consequence of Theorem 1.3 and Inequality (73). In fact, inequalities of the form [AM, (A.2)] (which generalize (73)) are intimately related to Hölder inequalities for non-commutative $L^{p}$-spaces. In the finite dimensional case, the non-commutative $L^{p}$-spaces correspond to spaces of Schatten class operators, as explained in Section 1.6.

\section{Corollary 1.5 (Universal determinant bounds)}

The universal determinant bound defined by (13) equals $\mathfrak{x}=1$.

Proof: $\quad$ Invoking Corollary 1.4, we deduce $\mathfrak{x} \leq 1$, see (13) and Definition 1.2. Now, let $\mathfrak{h}=\ell^{2}(\mathbb{N} ; \mathbb{C})$ with canonical ONB denoted by $\left\{e_{\mathfrak{i}}\right\}_{\mathfrak{i} \in \mathbb{N}}$. Take $\beta \in \mathbb{R}^{+}$, $\kappa=\mathbf{1}_{\mathbb{R}}, H=\lambda \mathbf{1}_{\mathfrak{h}}$ with $\lambda \in \mathbb{R}$ and $\mathfrak{M} \in \operatorname{Mat}(1, \mathbb{R})$ with $\mathfrak{M}_{1,1}=1$. Then, from Corollary 2.4 together with (18) and (36)-(37), for each $n \in 2 \mathbb{N}$ and all $N \in \mathbb{N}$, we directly compute that, for sufficiently large $n \gg 1$,

$$
\left|\operatorname{det}\left[\left\langle e_{k},\left(C_{\lambda \mathbf{1}_{\mathfrak{h}}} \hat{e}_{l}\right)(0)\right\rangle_{\mathfrak{h}}\right]_{k, l=1}^{N}\right|=\left(1-n^{-1} \beta \lambda\right)^{-N}\left(1+\left|1-n^{-1} \beta \lambda\right|^{-n}\right)^{-N} .
$$

In particular, for every $\varepsilon>0$ and $\beta \in \mathbb{R}^{+}$, there are $\lambda_{\varepsilon, \beta} \in \mathbb{R}$ and $n_{\varepsilon, \beta} \in \mathbb{N}$ such that, for all $n \geq n_{\varepsilon, \beta}$ and $N \in \mathbb{N}$,

$$
\left|\operatorname{det}\left[\left\langle e_{k},\left(C_{\lambda_{\varepsilon, \beta} \mathbf{1}_{\mathfrak{h}}} \hat{e}_{l}\right)(0)\right\rangle_{\mathfrak{h}}\right]_{k, l=1}^{N}\right| \geq(1-\varepsilon)^{2 N} \text {. }
$$

Using this lower bound and Corollary 1.4 , we then arrive at the equality $\mathfrak{x}=1$.

\subsection{Finite Dimensional Case and Hölder Inequalities for Schat- ten Norms}

As already discussed, we use Hölder inequalities for non-commutative $L^{p}$-spaces to derive determinant bounds (Definition 1.2). Here, we illustrate this approach in the finite dimensional case via Hölder inequalities for Schatten norms:

(i): Assume that $\mathfrak{h}$ is a finite dimensional Hilbert space. Then, the $C^{*}$-algebra $\overline{\mathrm{CA}} \mathrm{R}(\mathfrak{h} \otimes \mathbb{M})$ associated with $\mathfrak{h} \otimes \mathbb{M}$ can be identified with the space $\mathcal{B}(\mathbb{F})$ of all linear operators acting on the fermionic Fock space

$$
\mathbb{F} \doteq \wedge(\mathfrak{h} \otimes \mathbb{M})
$$


constructed from the one-particle Hilbert space $\mathfrak{h} \otimes \mathbb{M}$.

(ii): Take any faithful state $\rho$ on $\mathcal{B}(\mathbb{F})$ with cyclic representation $(\mathfrak{H}, \varkappa, \eta)$. By finite dimensionality, it follows that

$$
\varkappa(\mathrm{CAR}(\mathfrak{h} \otimes \mathbb{M}))^{\prime \prime}=\varkappa(\mathrm{CAR}(\mathfrak{h} \otimes \mathbb{M})) .
$$

Because $\rho$ is faithful, $\eta$ is separating for $\varkappa(\mathrm{CAR}(\mathfrak{h} \otimes \mathbb{M}))$ and the (TomitaTakesaki) modular objects associated with it are well-defined. Denote by $\Delta \in$ $\mathcal{B}(\mathfrak{H})$ the modular operator associated with the pair $(\varkappa(\mathrm{CAR}(\mathfrak{h} \otimes \mathbb{M})), \eta)$.

The cyclic representation $(\mathfrak{H}, \varkappa, \eta)$ is uniquely defined, up to a unitary transformation. It is explicitly given, for instance, by the so-called standard (cyclic) representation [DF, Section 5.4]: The space $\mathfrak{H}$ corresponds to the linear space $\mathcal{B}(\mathbb{F})$ endowed with the Hilbert-Schmidt scalar product

$$
\langle A, B\rangle_{\mathfrak{H}} \doteq \operatorname{Tr}_{\mathbb{F}}\left(A^{*} B\right), \quad A, B \in \mathfrak{H} .
$$

For any $A \in \mathcal{B}(\mathbb{F})$ we define the left and right multiplication operators $A$ and $A$ acting on $\mathcal{B}(\mathbb{F})$ by

$$
B \mapsto \underset{A}{\rightarrow} B \doteq A B \quad \text { and } \quad B \mapsto \notin A B \doteq B A
$$

respectively. The representation $\varkappa$ is the left multiplication, i.e.,

$$
\varkappa(A) \doteq \stackrel{A}{\rightarrow}, \quad A \in \mathcal{B}(\mathbb{F}) .
$$

The cyclic vector $\eta$ is defined by

$$
\eta \doteq \mathrm{D}^{1 / 2} \in \mathfrak{H}
$$

with $\mathrm{D} \in \mathcal{B}(\mathbb{F})$ being the unique positive operator such that

$$
\rho(A) \doteq \operatorname{Tr}_{\mathbb{F}}(\mathrm{D} A), \quad A \in \mathcal{B}(\mathbb{F}) .
$$

I.e., $\mathrm{D}$ is the density matrix of the state $\rho$. In this representation, the modular operator $\Delta$ associated with $\rho$ is equal to

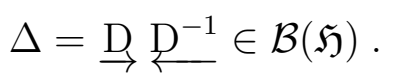

Note that if a state is faithful then its density matrix D is invertible. The $(\beta-$ rescaled) modular group is the one-parameter group $\sigma \equiv\left\{\sigma_{t}\right\}_{t \in \mathbb{R}}$ defined by

$$
\sigma_{t}(\stackrel{A}{\rightarrow}) \doteq \Delta^{-i t \beta^{-1}} \stackrel{A}{\rightarrow} \Delta^{i t \beta^{-1}} \quad A \in \mathcal{B}(\mathbb{F}) .
$$


(iii): Now, we fix $n \in 2 \mathbb{N}$ and apply this last construction to the quasi-free states $\bar{\rho}=\rho_{S_{\nu}}, \nu \in \mathbb{R}^{+}$, which are defined from symbols $S_{\nu}$ (18). See Section 2.1. Denote their standard representations by $\left(\mathfrak{H}_{\nu}, \varkappa_{\nu}, \eta_{\nu}\right)$, their density matrices by $\mathrm{D}_{\nu}$ and the associated modular operators by $\Delta_{\nu}$. We infer from (24), (25), (26), Corollary 2.4, the defining properties of Bogoliubov automorphisms (compare (27) with (67)-(69)), the cyclicity of traces, and the assumptions and definitions of Theorem 1.3 that

$$
\begin{gathered}
\operatorname{det}\left[\mathfrak{M}_{j_{k}, j_{N+l}}\left\langle\varphi_{N+l},\left(C_{H} \kappa(\hat{H}) \hat{\varphi}_{k}\right)\left(\alpha_{k}-\alpha_{N+l}\right)\right\rangle_{\mathfrak{h}}\right]_{k, l=1}^{N} \\
=\lim _{\nu \rightarrow \infty}(-1)^{\pi} \operatorname{Tr}_{\mathbb{F}}\left(\mathrm{D}_{\nu}^{\tilde{\alpha}_{\pi-1}(1) \beta^{-1}} \mathrm{D}_{\nu}^{\frac{1}{2}} x_{1}\left(\prod_{j=2}^{p-1}\left(\mathrm{D}_{\nu}^{\vartheta_{j}} x_{j}\right)\right) \mathrm{D}_{\nu}^{\frac{1}{2}-\beta^{-1} \tilde{\alpha}_{\pi^{-1}(p-1)}}\right. \\
\left.\mathrm{D}_{\nu}^{\beta^{-1} \tilde{\alpha}_{\pi^{-1}(p)}-\frac{1}{2}} x_{p}\left(\prod_{j=p+1}^{2 N}\left(\mathrm{D}_{\nu}^{\vartheta_{j}} x_{j}\right)\right) \mathrm{D}_{\nu}^{\frac{1}{2}} \mathrm{D}_{\nu}^{\left.-\beta^{-1} \tilde{\alpha}_{\pi^{-1}(2 N)}\right)}\right) \\
=\lim _{\nu \rightarrow \infty}(-1)^{\pi}\left\langle\Delta_{\nu}^{\frac{1}{2}-\beta^{-1} \tilde{\alpha}_{\pi^{-1}(p-1)}} x_{p-1}^{*} \Delta_{\nu}^{\vartheta_{p-1}} \cdots x_{2}^{*} \Delta_{\nu}^{\vartheta_{2}} x_{1}^{*} \eta_{\nu},\right. \\
\left.\Delta_{\nu}^{\beta^{-1} \tilde{\alpha}_{\pi}-1(p)}{ }^{-\frac{1}{2}} x_{p} \Delta_{\nu}^{\vartheta_{p+1}} x_{p+1} \cdots \Delta_{\nu}^{\vartheta_{N}} x_{N} \eta_{\nu}\right\rangle_{\mathfrak{H}_{\nu}},
\end{gathered}
$$

that is, Equation (14).

(iv): Schatten norms on $\mathcal{B}(\mathbb{F})$ are defined by

$$
\|A\|_{s} \doteq\left(\operatorname{Tr}_{\mathbb{F}}\left(|A|^{s}\right)\right)^{\frac{1}{s}}, \quad A \in \mathcal{B}(\mathbb{F}), s \geq 1
$$

and

$$
\|A\|_{\infty} \doteq \lim _{s \rightarrow \infty}\left(\operatorname{Tr}_{\mathbb{F}}\left(|A|^{s}\right)\right)^{\frac{1}{s}}=\|A\|_{\mathcal{B}(\mathbb{F})}, \quad A \in \mathcal{B}(\mathbb{F}) .
$$

Remark that the norm on the Hilbert space $\mathfrak{H}$ defined from the scalar product (24) is the Hilbert-Schmidt norm, i.e.,

$$
\|A\|_{\mathfrak{H}}=\|A\|_{2}, \quad A \in \mathcal{B}(\mathbb{F}) \equiv \mathfrak{H} .
$$

(v): Hölder inequalities for Schatten norms refer to the following bounds: For any $\bar{n} \in 2 \mathbb{N}, r, s_{1}, \ldots, s_{n} \in[1, \infty]$ such that $\sum_{j=1}^{n} 1 / s_{j}=1 / r$, and all operators 
$A_{1}, \ldots, A_{n} \in \mathcal{B}(\mathbb{F})$

$$
\left\|A_{1} \cdots A_{n}\right\|_{r} \leq \prod_{j=1}^{n}\left\|A_{j}\right\|_{s_{j}} .
$$

This type of inequality combined with (28) implies Corollary 1.4 in the finite dimensional case.

(vi): Indeed, for any integer $N \in \mathbb{N}$ and strictly positive parameter $\zeta \in \mathbb{R}^{+}$, define the tube

$$
\mathfrak{T}_{N}^{(\zeta)} \doteq\left\{\left(z_{1}, \ldots, z_{N}\right) \in \mathbb{C}^{N}: \forall j \in\{1, \ldots, N\}, \operatorname{Re}\left(z_{j}\right) \geq 0, \sum_{j=1}^{N} \operatorname{Re}\left(z_{j}\right) \leq \zeta\right\}
$$

Let $\rho$ be a faithful quasi-free state on $\mathcal{B}(\mathbb{F})$ and denote by $H_{\rho}=H_{\rho}^{*} \in \mathcal{B}(\mathfrak{h} \otimes \mathbb{M})$ the unique self-adjoint operator such that the symbol $S_{\rho}$ of $\rho$ equals

$$
S_{\rho}=\frac{1}{1+\mathrm{e}^{H_{\rho}}} .
$$

See beginning of Section 2.1 for more explanations on quasi-free states in relation with their symbols.

Choose $\Psi_{1}, \ldots, \Psi_{N} \in \mathfrak{h} \otimes \mathbb{M}$ and pick a family $\left\{a^{\#}\left(\Psi_{q}\right)\right\}_{q=1}^{N}$ of elements of $\operatorname{CAR}(\mathfrak{h} \otimes \mathbb{M})$, where the notation " $a$ " " stands for either " $a$ " " or " $a$ ". For any complex vector $\left(z_{1}, \ldots, z_{N}\right) \in \mathfrak{T}_{N}^{(1 / 2)}$, we observe from (26) that

$$
\begin{aligned}
& \Delta^{z_{1}} \varkappa\left(a^{\#}\left(\Psi_{1}\right)\right) \Delta^{z_{2}} \cdots \Delta^{z_{N}} \varkappa\left(a^{\#}\left(\Psi_{N}\right)\right) \eta \\
= & \mathrm{D}^{\operatorname{Re}\left(z_{1}\right)} a^{\#}\left(\mathrm{e}^{-i \operatorname{Im}\left(z_{1}\right) H_{\rho}} \Psi_{1}\right) \mathrm{D}^{\operatorname{Re}\left(z_{2}\right)} a^{\#}\left(\mathrm{e}^{-i\left(\operatorname{Im}\left(z_{1}\right)+\operatorname{Im}\left(z_{2}\right)\right) H_{\rho}} \Psi_{2}\right) \\
& \cdots \mathrm{D}^{\operatorname{Re}\left(z_{N}\right)} a^{\#}\left(\mathrm{e}^{-i\left(\operatorname{Im}\left(z_{1}\right)+\cdots+\operatorname{Im}\left(z_{N}\right)\right) H_{\rho}} \Psi_{N}\right) \mathrm{D}^{1 / 2-\left(\operatorname{Re}\left(z_{1}\right)+\cdots+\operatorname{Re}\left(z_{N}\right)\right)} .
\end{aligned}
$$

By applying Hölder inequalities (29) and (30), we obtain from the last equality that

$$
\begin{aligned}
& \left\|\Delta^{z_{1}} \varkappa\left(a^{\#}\left(\Psi_{1}\right)\right) \Delta^{z_{2}} \cdots \Delta^{z_{N}} \varkappa\left(a^{\#}\left(\Psi_{N}\right)\right) \eta\right\|_{\mathfrak{H}} \\
& \leq\left\|\mathrm{D}^{1 / 2-\left(\operatorname{Re}\left(z_{1}\right)+\cdots+\operatorname{Re}\left(z_{N}\right)\right)}\right\|_{\frac{1}{1 / 2-\left(\operatorname{Re}\left(z_{1}\right)+\cdots+\operatorname{Re}\left(z_{N}\right)\right)}} \\
& \quad \times \prod_{q=1}^{N}\left\|\mathrm{D}^{\operatorname{Re}\left(z_{q}\right)}\right\|_{\frac{1}{\operatorname{Re}\left(z_{q}\right)}}\left\|a^{\#}\left(\mathrm{e}^{i\left(\operatorname{Im}\left(z_{1}\right)+\cdots+\operatorname{Im}\left(z_{q}\right)\right) H_{\rho}} \Psi_{q}\right)\right\|_{\infty},
\end{aligned}
$$


which, combined with $\|\mathrm{D}\|_{1}=1$, in turn implies that

$$
\left\|\Delta^{z_{1}} \varkappa\left(a^{\#}\left(\Psi_{1}\right)\right) \Delta^{z_{2}} \cdots \Delta^{z_{N}} \varkappa\left(a^{\#}\left(\Psi_{N}\right)\right) \eta\right\|_{\mathfrak{H}} \leq \prod_{q=1}^{N}\left\|\Psi_{q}\right\|_{\mathfrak{h} \otimes \mathbb{M}} .
$$

This inequality corresponds to (73) in the finite dimensional case. Therefore, Equation (28) combined with Inequality (32) implies Corollary 1.4 when $\mathfrak{h}$ is a finite dimensional Hilbert space.

\section{Technical Proofs}

\subsection{Quasi-Free States}

Let $\mathcal{H}$ be some Hilbert space and $\operatorname{CAR}(\mathcal{H})$ the associated CAR $C^{*}$-algebra generated by the unit 1 and the family $\{a(\varphi)\}_{\varphi \in \mathcal{H}}$ of elements satisfying the canonical commutation relations (CAR): For any $\varphi_{1}, \varphi_{2} \in \mathcal{H}$,

$$
\begin{aligned}
a\left(\varphi_{1}\right) a\left(\varphi_{2}\right)+a\left(\varphi_{2}\right) a\left(\varphi_{1}\right) & =0, \\
a\left(\varphi_{1}\right)^{*} a\left(\varphi_{2}\right)+a\left(\varphi_{2}\right) a\left(\varphi_{1}\right)^{*} & =\left\langle\varphi_{2}, \varphi_{1}\right\rangle_{\mathcal{H}} \mathbf{1} .
\end{aligned}
$$

Strictly speaking, the above conditions only define $\operatorname{CAR}(\mathcal{H})$ up to an isomorphism of $C^{*}$-algebras. See, e.g., [BR2, Theorem 5.2.5]. As explained in Section 1.3 for the special case $\mathcal{H}=\mathfrak{h} \otimes \mathbb{M}$, the generator $a(\varphi) \in \mathrm{CAR}(\mathcal{H})$ is interpreted as the annihilation operator associated with $\varphi \in \mathcal{H}$ whereas its adjoint

$$
a^{+}(\varphi) \doteq a(\varphi)^{*}, \quad \varphi \in \mathcal{H},
$$

is the corresponding creation operator.

Here, quasi-free states on $\operatorname{CAR}(\mathcal{H})$ are positive linear functionals $\rho \in \operatorname{CAR}(\mathcal{H})^{*}$ such that $\rho(\mathbf{1})=1$ and, for all $N_{1}, N_{2} \in \mathbb{N}$ and $\varphi_{1}, \ldots, \varphi_{N_{1}+N_{2}} \in \mathcal{H}$,

$$
\rho\left(a^{+}\left(\varphi_{1}\right) \cdots a^{+}\left(\varphi_{N_{1}}\right) a\left(\varphi_{N_{1}+N_{2}}\right) \cdots a\left(\varphi_{N_{1}+1}\right)\right)=0
$$

if $N_{1} \neq N_{2}$, while in the case $N_{1}=N_{2} \equiv N$,

$$
\rho\left(a^{+}\left(\varphi_{1}\right) \cdots a^{+}\left(\varphi_{N}\right) a\left(\varphi_{2 N}\right) \cdots a\left(\varphi_{N+1}\right)\right)=\operatorname{det}\left[\rho\left(a^{+}\left(\varphi_{k}\right), a\left(\varphi_{N+l}\right)\right)\right]_{k, l=1}^{N} .
$$




\section{Remark 2.1 (Definitions of quasi-free states in the literature)}

Some authors relax Condition (35) in the definition of quasi-free states. Within this more general framework quasi-free states fulfilling (35) are then referred as gauge invariant quasi-free states of the corresponding CAR $C^{*}$-algebras. For instance, see [A, Definition 3.1]. Note indeed that [A, Definition 3.1, Condition (3.1)] only imposes on the quasi-free state to be even, but not necessarily gauge invariant.

The operator $S_{\rho} \in \mathcal{B}(\mathcal{H})$ defined from

$$
\left\langle\varphi_{2}, S_{\rho} \varphi_{1}\right\rangle_{\mathcal{H}}=\rho\left(a^{+}\left(\varphi_{1}\right) a\left(\varphi_{2}\right)\right), \quad \varphi_{1}, \varphi_{2} \in \mathcal{H}
$$

is named the symbol of the quasi-free state $\rho$. By the positivity and normalization of states, it follows that symbols are positive (self-adjoint) operators with spectrum lying on the unit interval $[0,1]$. Conversely, any such positive operator $S \leq \mathbf{1}_{\mathcal{H}}$ on $\mathcal{H}$ uniquely defines a quasi-free state $\rho_{S}$ on $\operatorname{CAR}(\mathcal{H})$ such that

$$
\rho_{S}\left(a^{+}\left(\varphi_{1}\right) a\left(\varphi_{2}\right)\right)=\left\langle\varphi_{2}, S \varphi_{1}\right\rangle_{\mathcal{H}}, \quad \varphi_{1}, \varphi_{2} \in \mathcal{H} .
$$

A monomial in the annihilation and creation operators is normally ordered if the creation operators appearing in the monomial are on the left side of all annihilation operators in the same monomial, like

$$
a^{+}\left(\varphi_{1}\right) \cdots a^{+}\left(\varphi_{N_{1}}\right) a\left(\varphi_{2 N_{1}}\right) \cdots a\left(\varphi_{N_{1}+1}\right) .
$$

By the above definition, if $\rho$ is a quasi-free state and $\mathcal{M} \in \mathrm{CAR}(\mathcal{H})$ is a normally ordered monomial in the annihilation and creation operators, then $\rho(\mathcal{M})$ is the determinant of a matrix, the entries of which are given by $\rho$ acting on monomials of degree two. We show below that this pivotal property of quasi-free states remains valid even if $\mathcal{M}$ is not normally ordered.

This is not surprising. For instance, [A, Definition 3.1, Condition (3.2)] also essentially says that if the state is quasi-free then expectation values (with respect to this state) of any monomial (not necessarily normally ordered) of arbitrary even degree is a determinant of a matrix, the entries of which are expectation values of monomials of degree two. However, beyond this fact, we would like to give the explicit behavior of such expectation values with respect to arbitrary permutations of creation and annihilation operators in large monomials. This point is crucial here and is given by Lemma 2.2.

To this end, we introduce some notation. If $\pi$ is a permutation of $n \in \mathbb{N}$ elements (i.e., a bijective function from $\{1, \ldots, n\}$ to $\{1, \ldots, n\}$ ) with sign $(-1)^{\pi}$, 
we define the monomial $\mathbb{O}_{\pi}\left(A_{1}, \ldots, A_{n}\right) \in \operatorname{CAR}(\mathcal{H})$ in $A_{1}, \ldots, A_{n} \in \operatorname{CAR}(\mathcal{H})$ by the product

$$
\mathbb{O}_{\pi}\left(A_{1}, \ldots, A_{n}\right) \doteq(-1)^{\pi} A_{\pi^{-1}(1)} \cdots A_{\pi^{-1}(n)} .
$$

In other words, $\mathbb{O}_{\pi}$ places the operator $A_{k}$ at the $\pi(k)$ th position in the monomial $(-1)^{\pi} A_{\pi^{-1}(1)} \cdots A_{\pi^{-1}(n)}$. Further, for all $k, l \in\{1, \ldots, n\}, k \neq l$,

$$
\pi_{k, l}:\{1,2\} \rightarrow\{1,2\}
$$

is the identity function if $\pi(k)<\pi(l)$, otherwise $\pi_{k, l}$ interchanges 1 and 2. Then, the following property of quasi-free states holds true:

\section{Lemma 2.2 (Quasi-free states on general monomials)}

Let $\rho$ be a quasi-free state on the $C^{*}$-algebra $\operatorname{CAR}(\mathcal{H})$. For any $N_{1}, N_{2} \in \mathbb{N}$, all permutations $\pi$ of $N_{1}+N_{2}$ elements and $\varphi_{1}, \ldots, \varphi_{N_{1}+N_{2}} \in \mathcal{H}$,

$$
\rho\left(\mathbb{O}_{\pi}\left(a^{+}\left(\varphi_{1}\right), \ldots, a^{+}\left(\varphi_{N_{1}}\right), a\left(\varphi_{N_{1}+N_{2}}\right), \ldots, a\left(\varphi_{N_{1}+1}\right)\right)\right)=0
$$

if $N_{1} \neq N_{2}$, while in the case $N_{1}=N_{2} \equiv N$,

$$
\begin{aligned}
& \rho\left(\mathbb{O}_{\pi}\left(a^{+}\left(\varphi_{1}\right), \ldots, a^{+}\left(\varphi_{N}\right), a\left(\varphi_{2 N}\right), \ldots, a\left(\varphi_{N+1}\right)\right)\right) \\
= & \operatorname{det}\left[\rho\left(\mathbb{O}_{\pi_{k, N+l}}\left(a^{+}\left(\varphi_{k}\right), a\left(\varphi_{N+l}\right)\right)\right)\right]_{k, l=1}^{N} .
\end{aligned}
$$

Proof: $\quad$ By (33) and (34), if the monomial

$$
\mathbb{O}_{\pi}\left(a^{+}\left(\varphi_{1}\right), \ldots, a^{+}\left(\varphi_{N_{1}}\right), a\left(\varphi_{N_{1}+N_{2}}\right), \ldots, a\left(\varphi_{N_{1}+1}\right)\right)
$$

contains different numbers of annihilation and creation operators (i.e., $N_{1} \neq N_{2}$ ), then it can be written as a sum of normally ordered monomials with the same property. By (35) and the linearity of states, we thus deduce (41).

We consider the case $N_{1}=N_{2} \equiv N \in \mathbb{N}$. Assertion (42) trivially holds if $N=1$ and we can assume from now on that $N \geq 2$.

For convenience, the notation " $a$ " " stands for either " $a$ " or " $a$ ". In particular, we write the monomial

$$
\mathbb{O}_{\pi}\left(a^{+}\left(\varphi_{1}\right), \ldots, a^{+}\left(\varphi_{n}\right), a\left(\varphi_{2 N}\right), \ldots, a\left(\varphi_{N+1}\right)\right)=(-1)^{\pi} a_{1}^{\#} \cdots a_{2 N}^{\#} .
$$


Let

$$
\begin{aligned}
& k_{\pi} \doteq \min \{\pi(N+1), \ldots, \pi(2 N)\} \leq N+1, \\
& k_{\pi}^{+} \doteq \max \{\pi(1), \ldots, \pi(N)\} \geq N .
\end{aligned}
$$

The parameter $k_{\pi}$ is the position the first annihilation operator appearing in the monomial $a_{1}^{\#} \cdots a_{2 N}^{\#}$ while $k_{\pi}^{+}$is the position of the last creation operator appearing in $a_{1}^{\#} \cdots a_{2 N}^{\#}$. In other words,

$$
\begin{aligned}
& \mathbb{O}_{\pi}\left(a^{+}\left(\varphi_{1}\right), \ldots, a^{+}\left(\varphi_{N}\right), a\left(\varphi_{2 N}\right), \ldots, a\left(\varphi_{N+1}\right)\right) \\
= & (-1)^{\pi} a_{1}^{+} \cdots a_{k_{\pi}-1}^{+} a_{k_{\pi}} a_{k_{\pi}+1}^{\#} \cdots a_{k_{\pi}^{+}-1}^{\#} a_{k_{\pi}^{+}}^{+} a_{k_{\pi}^{+}+1} \cdots a_{2 N} .
\end{aligned}
$$

Note that $k_{\pi}=N+1$ iff the monomial is normally ordered. The same holds true if $k_{\pi}^{+}=N$. In particular, $k_{\pi}=N+1$ iff $k_{\pi}^{+}=N$. We will prove Assertion (42) by induction in the parameter

$$
N_{\pi} \doteq k_{\pi}^{+}-k_{\pi}+1 \geq 0
$$

Observe that $N_{\pi}=0$ iff the monomial is normally ordered and Assertion (42) holds in this case because of (33), (36) and the antisymmetry of the determinant under permutations of its lines or rows.

Assume now that $N_{\pi} \geq 1$. Thus, $k_{\pi} \leq N$ and $k_{\pi}^{+} \geq N+1$. If $k_{\pi}>2$ and $2 N-k_{\pi}>3$ then

$$
\begin{aligned}
& (-1)^{\pi} \mathbb{O}_{\pi}\left(a^{+}\left(\varphi_{1}\right), \ldots, a^{+}\left(\varphi_{N}\right), a\left(\varphi_{2 N}\right), \ldots, a\left(\varphi_{N+1}\right)\right) \\
= & a_{1}^{+} \cdots a_{k_{\pi}-1}^{+}\left(a_{k_{\pi}} a_{k_{\pi}+1}^{\#}+a_{k_{\pi}+1}^{\#} a_{k_{\pi}}\right) a_{k_{\pi}+2}^{\#} \cdots a_{2 N}^{\#} \\
& -a_{1}^{+} \cdots a_{k_{\pi}-1}^{+} a_{k_{\pi}+1}^{\#}\left(a_{k_{\pi}} a_{k_{\pi}+2}^{\#}+a_{k_{\pi}+2}^{\#} a_{k_{\pi}}\right) a_{k_{\pi}+3}^{\#} \cdots a_{2 N}^{\#} \\
& +a_{1}^{+} \cdots a_{k_{\pi}-1}^{+} \sum_{l=3}^{2 N-k_{\pi}-2}(-1)^{l-1} a_{k_{\pi}+1}^{\#} \cdots a_{k_{\pi}+l-1}^{\#}\left(a_{k_{\pi}} a_{k_{\pi}+l}^{\#}\right. \\
& \left.+a_{k_{\pi}+l}^{\#} a_{k_{\pi}}\right) a_{k_{\pi}+l+1}^{\#} \cdots a_{2 N}^{\#} \\
& +(-1)^{2 N-k_{\pi}-2} a_{1}^{+} \cdots a_{k_{\pi}-1}^{+} a_{k_{\pi}+1}^{\#} \cdots a_{2 N-2}^{\#}\left(a_{k_{\pi}} a_{2 N-1}^{\#}+a_{2 N-1}^{\#} a_{k_{\pi}}\right) a_{2 N}^{\#} \\
& +(-1)^{2 N-k_{\pi}-1} a_{1}^{+} \cdots a_{k_{\pi}-1}^{+} a_{k_{\pi}+1}^{\#} \cdots a_{2 N-1}^{\#}\left(a_{k_{\pi}} a_{2 N}^{\#}+a_{2 N}^{\#} a_{k_{\pi}}\right) \\
& +(-1)^{2 N-k_{\pi}} a_{1}^{+} \cdots a_{k_{\pi}-1}^{+} a_{k_{\pi}+1}^{\#} \cdots a_{2 N}^{\#} a_{k_{\pi}} .
\end{aligned}
$$

Mutatis mutandis if $k_{\pi}=1,2$ or $2 N-k_{\pi}=2,3$. It is convenient to use the definition

$$
q_{\pi} \doteq 2 N-\pi^{-1}\left(k_{\pi}\right)+1 \in\{1, \ldots, N\}
$$


which implies $a_{k_{\pi}}=a\left(\varphi_{N+q_{\pi}}\right)$. By combining (43) with the CAR (33) and (34), we deduce the equality

$$
\begin{aligned}
& (-1)^{\pi} \mathbb{O}_{\pi}\left(a^{+}\left(\varphi_{1}\right), \ldots, a^{+}\left(\varphi_{N}\right), a\left(\varphi_{2 N}\right), \ldots, a\left(\varphi_{N+1}\right)\right) \\
= & (-1)^{2 N-k_{\pi}} a_{1}^{+} \cdots a_{k_{\pi}-1}^{+} a_{k_{\pi}+1}^{\#} \cdots a_{2 N}^{\#} a_{k_{\pi}} \\
& +\sum_{k=1}^{N}\left\langle\varphi_{N+q_{\pi}}, \varphi_{k}\right\rangle_{\mathcal{H}}\left\{\mathbf{1}\left[k_{\pi}+1=\pi(k)\right] a_{1}^{+} \cdots a_{k_{\pi}-1}^{+} a_{k_{\pi}+2}^{\#} \cdots a_{2 N}^{\#}\right. \\
& -\mathbf{1}\left[k_{\pi}+2=\pi(k)\right] a_{1}^{+} \cdots a_{k_{\pi}-1}^{+} a_{k_{\pi}+1}^{\#} a_{k_{\pi}+3}^{\#} \cdots a_{2 N}^{\#} \\
& +\mathbf{1}\left[2 N-1>\pi(k)>k_{\pi}+2\right](-1)^{\pi(k)-k_{\pi}-1} \\
& a_{1}^{+} \cdots a_{k_{\pi}-1}^{+} a_{k_{\pi}+1}^{\#} \cdots a_{\pi(k)-1}^{\#} a_{\pi(k)+1}^{\#} \cdots a_{2 N}^{\#} \\
& +\mathbf{1}[2 N-1=\pi(k)](-1)^{2 N-k_{\pi}-2} a_{1}^{+} \cdots a_{k_{\pi}-1}^{+} a_{k_{\pi}+1}^{\#} \cdots a_{2 N-2}^{\#} a_{2 N}^{\#} \\
& \left.+\mathbf{1}[2 N=\pi(k)](-1)^{2 N-k_{\pi}-1} a_{1}^{+} \cdots a_{k_{\pi}-1}^{+} a_{k_{\pi}+1}^{\#} \cdots a_{2 N-1}^{\#}\right\}
\end{aligned}
$$

when $k_{\pi}>2$ and $2 N-k_{\pi}>3$. Mutatis mutandis if $k_{\pi}=1,2$ or $2 N-k_{\pi}=2,3$. For any $k \in\{1, \ldots, N\}$, we fix a permutation $\pi^{(k)}$ of $2(N-1)$ elements such that

$$
\begin{aligned}
& \mathbb{O}_{\pi^{(k)}}\left(a^{+}\left(\varphi_{1}\right), \ldots,\right. a^{+}\left(\varphi_{k-1}\right), a^{+}\left(\varphi_{k+1}\right), \ldots, a^{+}\left(\varphi_{N}\right), \\
&\left.a\left(\varphi_{2 N}\right), \ldots, a\left(\varphi_{N+q_{\pi}+1}\right), a\left(\varphi_{N+q_{\pi}-1}\right), \ldots, a\left(\varphi_{N+1}\right)\right) \\
&=(-1)^{\pi^{(k)}} a_{1}^{+} \cdots a_{k_{\pi}-1}^{+} a_{k_{\pi}+1}^{\#} \cdots a_{\pi(k)-1}^{\#} a_{\pi(k)+1}^{\#} \cdots a_{2 N}^{\#} .
\end{aligned}
$$

(Recall that $N \geq 2$ is assumed without loss of generality.) Similarly, $\tilde{\pi}$ is a permutation of $2 N$ elements such that

$$
\begin{aligned}
\mathbb{O}_{\tilde{\pi}}\left(a^{+}\left(\varphi_{1}\right), \ldots, a^{+}\left(\varphi_{N}\right), a\left(\varphi_{2 N}\right), \ldots\right. & \left., a\left(\varphi_{N+1}\right)\right) \\
= & (-1)^{\tilde{\pi}} a_{1}^{+} \cdots a_{k_{\pi}-1}^{+} a_{k_{\pi}+1}^{\#} \cdots a_{2 N}^{\#} a_{k_{\pi}} .
\end{aligned}
$$

By using this notation, we rewrite (44) as

$$
\begin{gathered}
(-1)^{\pi} \mathbb{O}_{\pi}\left(a^{+}\left(\varphi_{1}\right), \ldots, a^{+}\left(\varphi_{N}\right), a\left(\varphi_{2 N}\right), \ldots, a\left(\varphi_{N+1}\right)\right) \\
=(-1)^{k_{\pi}}(-1)^{\tilde{\pi}} \mathbb{O}_{\tilde{\pi}}\left(a^{+}\left(\varphi_{1}\right), \ldots, a^{+}\left(\varphi_{N}\right), a\left(\varphi_{2 N}\right), \ldots, a\left(\varphi_{N+1}\right)\right) \\
+\sum_{k=1}^{N} \mathbf{1}\left[\pi(k)>k_{\pi}\right](-1)^{\pi(k)-k_{\pi}-1}\left\langle\varphi_{N+q_{\pi}}, \varphi_{k}\right\rangle_{\mathcal{H}}(-1)^{\pi^{(k)}} \\
\times \mathbb{O}_{\pi^{(k)}}\left(a^{+}\left(\varphi_{1}\right), \ldots, a^{+}\left(\varphi_{k-1}\right), a^{+}\left(\varphi_{k+1}\right), \ldots, a^{+}\left(\varphi_{N}\right), a\left(\varphi_{2 N}\right),\right. \\
\left.\ldots, a\left(\varphi_{N+q_{\pi}+1}\right), a\left(\varphi_{N+q_{\pi}-1}\right), \ldots, a\left(\varphi_{N+1}\right)\right) .
\end{gathered}
$$


For all $k \in\{1, \ldots, N\}$, note that

$$
k_{\pi^{(k)}}^{+} \leq k_{\pi}^{+}-2 \quad \text { and } \quad k_{\pi^{(k)}} \geq k_{\pi} .
$$

As a consequence, for any $k \in\{1, \ldots, N\}$, the induction parameter $N_{\pi^{(k)}}$ associated with the permutation $\pi^{(k)}$ satisfies:

$$
N_{\pi^{(k)}} \doteq k_{\pi^{(k)}}^{+}-k_{\pi^{(k)}}+1 \leq k_{\pi}^{+}-k_{\pi}-1=N_{\pi}-2 .
$$

Similarly,

$$
k_{\tilde{\pi}}^{+}=k_{\pi}^{+}-1 \quad \text { and } \quad k_{\tilde{\pi}} \geq k_{\pi},
$$

which in turn imply

$$
N_{\tilde{\pi}} \doteq k_{\tilde{\pi}}^{+}-k_{\tilde{\pi}}+1 \leq k_{\pi}^{+}-k_{\pi}=N_{\pi}-1 .
$$

Observe furthermore that, for any $k \in\{1, \ldots, N\}$ such that $\pi(k)>k_{\pi}$,

$$
(-1)^{\tilde{\pi}}=(-1)^{\pi}(-1)^{k_{\pi}} \quad \text { and } \quad(-1)^{\pi^{(k)}}=(-1)^{\pi}(-1)^{q_{\pi}+k+k_{\pi}+\pi(k)} .
$$

Therefore, by using (48) together with (45), we arrive at the equality

$$
\begin{aligned}
& \mathbb{O}_{\pi}\left(a^{+}\left(\varphi_{1}\right), \ldots, a^{+}\left(\varphi_{N}\right), a\left(\varphi_{2 N}\right), \ldots, a\left(\varphi_{N+1}\right)\right) \\
&= \mathbb{O}_{\tilde{\pi}}\left(a^{+}\left(\varphi_{1}\right), \ldots, a^{+}\left(\varphi_{N}\right), a\left(\varphi_{2 N}\right), \ldots, a\left(\varphi_{N+1}\right)\right) \\
&-\sum_{k=1}^{N} 1\left[\pi(k)>k_{\pi}\right](-1)^{q_{\pi}+k}\left\langle\varphi_{N+q_{\pi}}, \varphi_{k}\right\rangle_{\mathcal{H}} \\
& \mathbb{O}_{\pi^{(k)}}\left(a^{+}\left(\varphi_{1}\right), \ldots, a^{+}\left(\varphi_{k-1}\right), a^{+}\left(\varphi_{k+1}\right), \ldots, a^{+}\left(\varphi_{N}\right), a\left(\varphi_{2 N}\right),\right. \\
&\left.\ldots, a\left(\varphi_{N+q_{\pi}+1}\right), a\left(\varphi_{N+q_{\pi}-1}\right), \ldots, a\left(\varphi_{N+1}\right)\right) .
\end{aligned}
$$

We use now the following definitions: For any $k, l \in\{1, \ldots, N\}$, the coefficients

$$
\begin{aligned}
& \mathrm{M}_{k, l} \doteq \rho\left(\mathbb{O}_{\pi_{k, N+l}}\left(a^{+}\left(\varphi_{k}\right), a\left(\varphi_{N+l}\right)\right)\right), \\
& \tilde{\mathrm{M}}_{k, l} \doteq \rho\left(\mathbb{O}_{\tilde{\pi}_{k, N+l}}\left(a^{+}\left(\varphi_{k}\right), a\left(\varphi_{N+l}\right)\right)\right),
\end{aligned}
$$

$k, l \in\{1, \ldots, N\}$, are the entries of two matrices $\mathrm{M}$ and $\tilde{\mathrm{M}}$, respectively. Let

$$
\mathrm{M}^{(k, l)} \doteq \operatorname{det}\left(\left[\mathrm{M}_{i, j}\right]_{i, j \in\{1, \ldots, N\}, i \neq k, j \neq l}\right)
$$


be the $k, l \in\{1, \ldots, N\}$ minor of $\mathrm{M}$, that is, the determinant of the $(N-1) \times(N-$ 1 ) matrix that results from deleting the $k$ th row and the $l$ th column of M. From the Laplace expansion for determinants (sometimes called cofactor expansion),

$$
\begin{aligned}
& \operatorname{det} \mathrm{M}=\sum_{k=1}^{N}(-1)^{q_{\pi}+k} \rho\left(\mathbb{O}_{\pi_{k, N+l}}\left(a^{+}\left(\varphi_{k}\right), a\left(\varphi_{N+q_{\pi}}\right)\right)\right) \mathrm{M}^{\left(k, q_{\pi}\right)} \\
& \operatorname{det} \tilde{\mathrm{M}}=\sum_{k=1}^{N}(-1)^{q_{\pi}+k} \rho\left(a^{+}\left(\varphi_{k}\right) a\left(\varphi_{N+q_{\pi}}\right)\right) \mathrm{M}^{\left(k, q_{\pi}\right)} .
\end{aligned}
$$

To derive the equality (51) we also use that $\tilde{\pi}_{k, N+l}=\pi_{k, N+l}$ whenever $l \neq q_{\pi}$, whereas it is the identity of the set $\{1,2\}$ for $l=q_{\pi}$. On the other hand, using (46)-(47) and the induction hypothesis for all $\tilde{N}_{\pi} \geq 0$ with $\tilde{N}_{\pi}<N_{\pi}$, we deduce that

$$
\begin{array}{r}
\mathrm{M}^{\left(k, q_{\pi}\right)}=\rho\left(\mathbb { O } _ { \pi ^ { ( k ) } } \left(a^{+}\left(\varphi_{1}\right), \ldots, a^{+}\left(\varphi_{k-1}\right), a^{+}\left(\varphi_{k+1}\right), \ldots, a^{+}\left(\varphi_{N}\right), a\left(\varphi_{2 N}\right),\right.\right. \\
\left.\left.\ldots, a\left(\varphi_{N+q_{\pi}+1}\right), a\left(\varphi_{N+q_{\pi}-1}\right), \ldots, a\left(\varphi_{N+1}\right)\right)\right)
\end{array}
$$

and

$$
\operatorname{det} \tilde{\mathrm{M}}=\rho\left(\mathbb{O}_{\tilde{\pi}}\left(a^{+}\left(\varphi_{1}\right), \ldots, a^{+}\left(\varphi_{N}\right), a\left(\varphi_{2 N}\right), \ldots, a\left(\varphi_{N+1}\right)\right)\right) .
$$

Thus, by induction, it follows from (34), (49), (50) and (51) that

$$
\begin{aligned}
& \rho\left(\mathbb{O}_{\pi}\left(a^{+}\left(\varphi_{1}\right), \ldots, a^{+}\left(\varphi_{N}\right), a\left(\varphi_{2 N}\right), \ldots, a\left(\varphi_{N+1}\right)\right)\right) \\
= & \sum_{k=1}^{N}(-1)^{q_{\pi}+k}\left\{\rho\left(a^{+}\left(\varphi_{k}\right) a\left(\varphi_{N+q_{\pi}}\right)\right)-\mathbf{1}\left[\pi(k)>k_{\pi}\right]\left\langle\varphi_{N+q_{\pi}}, \varphi_{k}\right\rangle_{\mathcal{H}}\right\} \mathrm{M}^{\left(k, q_{\pi}\right)} \\
= & \sum_{k=1}^{N}(-1)^{q_{\pi}+k} \rho\left(\mathbb{O}_{\pi_{k, N+q_{\pi}}}\left(a^{+}\left(\varphi_{k}\right), a\left(\varphi_{N+q_{\pi}}\right)\right)\right) \mathrm{M}^{\left(k, q_{\pi}\right)}=\operatorname{det} \mathrm{M} .
\end{aligned}
$$

\subsection{Representation of Discrete Time Covariance by Quasi-Free States}

(i): Take a Hilbert space $\mathfrak{h}$. To avoid technical issues, we assume for convenience that $\mathfrak{h}$ is separable. Pick next any (possibly unbounded) self-adjoint operator $H=$ 
$H^{*}$ acting on $\mathfrak{h}$. By the spectral theorem, there is a ( $\sigma$-finite) measure space $\left(\Omega_{H}, \mathfrak{A}_{H}, \mu_{H}\right)$, a unitary map $U_{H}$ from $\mathfrak{h}$ to $L^{2}\left(\Omega_{H} ; \mathbb{C}\right)$ and a $\mathfrak{A}_{H}$-measurable function $\lambda_{H}: \Omega_{H} \rightarrow \mathbb{R}$ such that

$$
U_{H} H U_{H}^{*}=\mathrm{m}_{\lambda_{H}},
$$

where $\mathrm{m}_{\lambda_{H}}$ is the multiplication operator on $L^{2}\left(\Omega_{H}\right)$ with the function $\lambda_{H}$. Using the unitary $U_{H}$ we can identify $\ell_{\text {ap }}^{2}\left(\mathbb{T}_{n} ; \mathfrak{h}\right)$ with $\ell_{\text {ap }}^{2}\left(\mathbb{T}_{n} ; L^{2}\left(\Omega_{H} ; \mathbb{C}\right)\right)$, i.e.,

$$
\hat{U}_{H} \ell_{\text {ap }}^{2}\left(\mathbb{T}_{n} ; \mathfrak{h}\right)=\ell_{\text {ap }}^{2}\left(\mathbb{T}_{n} ; L^{2}\left(\Omega_{H} ; \mathbb{C}\right)\right) .
$$

The latter, in turn, is canonically identified with

$$
\int_{\Omega_{H}}^{\oplus} \ell_{\mathrm{ap}}^{2}\left(\mathbb{T}_{n} ; \mathbb{C}\right) \mu_{H}(\mathrm{~d} \mathfrak{a}) \equiv L^{2}\left(\Omega_{H} ; \ell_{\mathrm{ap}}^{2}\left(\mathbb{T}_{n} ; \mathbb{C}\right)\right)
$$

In other words, by using $U_{H}$, we identify $\ell_{\text {ap }}^{2}\left(\mathbb{T}_{n} ; \mathfrak{h}\right)$ with (53). Note that the above direct integral is well-defined because $\ell_{\mathrm{ap}}^{2}\left(\mathbb{T}_{n} ; \mathbb{C}\right)$ is finite dimensional and $\left(\Omega_{H}, \mathfrak{A}_{H}, \mu_{H}\right)$ is a $\sigma$-finite measure space, since $\mathfrak{h}$ is assumed, for simplicity, to be separable.

(ii): With this convention, we obtain the relation

$$
\hat{U}_{H} \hat{H} \hat{U}_{H}^{*}=\int_{\Omega_{H}}^{\oplus} \lambda_{H}(\mathfrak{a}) \mathbf{1}_{\ell_{\mathrm{ap}}^{2}\left(\mathbb{T}_{n} ; \mathbb{C}\right)} \mu_{H}(\mathrm{~d} \mathfrak{a})
$$

The discrete derivative $\partial$ defined by (8) is meanwhile written in the new Hilbert space as

$$
\hat{U}_{H} \partial \hat{U}_{H}^{*}=\int_{\Omega_{H}}^{\oplus} \mathfrak{d} \mu_{H}(\mathrm{~d} \mathfrak{a})
$$

where $\mathfrak{d} \in \mathcal{B}\left(\ell_{\text {ap }}^{2}\left(\mathbb{T}_{n} ; \mathbb{C}\right)\right)$ is defined by

$$
\mathfrak{d} f(\alpha) \doteq \beta^{-1} n\left(f\left(\alpha+n^{-1} \beta\right)-f(\alpha)\right), \quad f \in \ell_{\mathrm{ap}}^{2}\left(\mathbb{T}_{n} ; \mathbb{C}\right), \alpha \in \mathbb{T}_{n}
$$

In particular, the discrete time covariance $C_{H}$, defined by (9), can be represented as

$$
\hat{U}_{H} C_{H} \hat{U}_{H}^{*}=-2 \int_{\Omega_{H}}^{\oplus} R\left(\mathfrak{d}, \lambda_{H}(\mathfrak{a})\right) \mu_{H}(\mathrm{~d} \mathfrak{a}),
$$

where $R(\mathfrak{d}, \lambda) \in \mathcal{B}\left(\ell_{\text {ap }}^{2}\left(\mathbb{T}_{n} ; \mathbb{C}\right)\right)$ is the resolvent

$$
R(\mathfrak{d}, \lambda) \doteq\left(\mathfrak{d}+\lambda \mathbf{1}_{\ell_{\mathrm{ap}}^{2}\left(\mathbb{T}_{n} ; \mathbb{C}\right)}\right)^{-1}, \quad \lambda \in \mathbb{R}
$$


By using the discrete delta function $\delta_{\mathrm{ap}} \in \ell_{\mathrm{ap}}^{2}\left(\mathbb{T}_{n} ; \mathbb{C}\right)$ defined by (2) and the convolution (6) together with direct computations, one easily checks that, for every $\beta \in \mathbb{R}^{+}, \lambda \in \mathbb{R}$ and any $n \in 2 \mathbb{N}$,

$$
-2 R(\mathfrak{d}, \lambda) f=g_{\lambda} * f, \quad f \in \ell_{\mathrm{ap}}^{2}\left(\mathbb{T}_{n} ; \mathbb{C}\right),
$$

with $g_{\lambda} \in \ell_{\text {ap }}^{2}\left(\mathbb{T}_{n} ; \mathbb{C}\right)$ being equal to (22)-(23) on $\mathbb{T}_{n} \cap(-\beta, 0]$.

(iii): We pick a (possibly unbounded) self-adjoint operator $H=H^{*}$ acting on $\mathfrak{h}$ and fix from now on $n \in 2 \mathbb{N}$. Then, because of (22), (23), (54) and (55), for any fixed $\beta, \nu \in \mathbb{R}^{+}$we introduce the unitary operator

$$
\mathrm{E} \doteq \operatorname{sgn}\left(\mathbf{1}_{\mathfrak{h}}-n^{-1} \beta H\right) \in \mathcal{B}(\mathfrak{h})
$$

and the (possibly unbounded) operator $H_{\nu} \doteq \digamma_{\nu}(H)$, see (15) and (16). For any $\nu \in \mathbb{R}$, the Hamiltonian $H_{\nu}$ gives rise to the symbol $S_{\nu}$ (18), which, as explained in Section 2.1, in turn yields a faithful quasi-free state $\rho_{S_{\nu}}$, with symbol $S_{\nu}>0$, on the $\mathrm{CAR} C^{*}$-algebra $\mathrm{CAR}(\mathfrak{h} \otimes \mathbb{M})$.

Let

$$
\mathfrak{D} \doteq \bigcup_{D \in \mathbb{R}^{+}} \operatorname{ran}\left(\mathbf{1}\left[-D \leq H_{\nu} \leq D\right]\right), \quad \nu \in \mathbb{R} .
$$

By the spectral theorem, it is a dense subspace of entire analytic vectors of $H_{\nu}$. Note additionally that $\mathfrak{D}$ does not depend on $\nu \in \mathbb{R}$.

(iv): Finally, similar to the permutation (40), for all $\alpha_{1}, \alpha_{2} \in \mathbb{T}_{n} \cap[0, \beta)$, we define the permutation

$$
\pi_{\alpha_{1}, \alpha_{2}}:\{1,2\} \rightarrow\{1,2\}
$$

as the identity map if $\alpha_{1} \leq \alpha_{2}$, while $\pi_{\alpha_{1}, \alpha_{2}}$ interchanges 1 and 2 when $\alpha_{1}>\alpha_{2}$. Then, quasi-free states $\rho_{S_{\nu}}, \nu \in \mathbb{R}^{+}$, give rise to the following representation of the discrete time covariance:

\section{Lemma 2.3 (Representation of the covariance by a quasi-free state)}

Let $\mathfrak{h}$ be any separable Hilbert space. Fix $\beta \in \mathbb{R}^{+}$, a self-adjoint operator $H=H^{*}$ acting on $\mathfrak{h}$, and $n \in 2 \mathbb{N}$. Then, for each bounded measurable positive function $\kappa$ from $\mathbb{R}$ to $\mathbb{R}_{0}^{+}$, all $m \in \mathbb{N}$, non-vanishing $\mathfrak{M} \in \operatorname{Mat}(m, \mathbb{R})$ with $\mathfrak{M} \geq 0, \alpha_{1}, \alpha_{2} \in \mathbb{T}_{n} \cap[0, \beta), \varphi_{1}, \varphi_{2} \in \mathfrak{D}$ and $j_{1}, j_{2} \in\{1, \ldots, m\}$,

$$
\begin{aligned}
\mathfrak{M}_{j_{1}, j_{2}}\left\langle\varphi_{2},\right. & \left.\left(C_{H} \kappa(\hat{H}) \hat{\varphi}_{1}\right)\left(\alpha_{1}-\alpha_{2}\right)\right\rangle_{\mathfrak{h}} \\
=\lim _{\nu \rightarrow \infty} \rho_{S_{\nu}}\left(\mathbb { O } _ { \pi _ { \alpha _ { 1 } , \alpha _ { 2 } } } \left(a^{+}\right.\right. & \left(\left(\mathrm{e}^{-\alpha_{1} H_{\nu}} \mathrm{E}^{-\beta^{-1} n \alpha_{1}} \varphi_{1}\right) \otimes \mathfrak{e}_{j_{1}}\right), \\
& \left.\left.a\left(\left(\mathrm{e}^{\left(\alpha_{2}+n^{-1} \beta\right) H_{\nu}} \mathrm{E}^{\beta^{-1} n \alpha_{2}+1} \varphi_{2}\right) \otimes \mathfrak{e}_{j_{2}}\right)\right)\right)
\end{aligned}
$$


with $\mathfrak{e}_{j} \doteq\left[e_{j}\right] \in \mathbb{M}$ being the vectors of $\mathbb{M}$ satisfying (11) and where $\mathbb{O}_{\pi_{\alpha_{1}, \alpha_{2}}}$ is defined by (39) for $\pi=\pi_{\alpha_{1}, \alpha_{2}}$.

Proof: Fix all the parameters of the lemma. Note that

$$
\begin{aligned}
& \mathfrak{M}_{j_{1}, j_{2}}\left\langle\varphi_{2},\left(C_{H} \kappa(\hat{H}) \hat{\varphi}_{1}\right)\left(\alpha_{1}-\alpha_{2}\right)\right\rangle_{\mathfrak{h}} \\
= & \mathfrak{M}_{j_{1}, j_{2}}\left\langle\kappa(\hat{H})^{1 / 2} \varphi_{2},\left(C_{H} \kappa(\hat{H})^{1 / 2} \hat{\varphi}_{1}\right)\left(\alpha_{1}-\alpha_{2}\right)\right\rangle_{\mathfrak{h}} .
\end{aligned}
$$

Therefore, we can assume without loss of generality that $\kappa=\mathbf{1}_{\mathbb{R}}$.

We deduce from Equations (54) and (55) that

$$
\begin{aligned}
& \left\langle\varphi_{2},\left(C_{H} \hat{\varphi}_{1}\right)\left(\alpha_{1}-\alpha_{2}\right)\right\rangle_{\mathfrak{h}} \\
= & \left\langle\psi_{2},\left(\int_{\Omega_{H}}^{\oplus} g_{\lambda_{H}(\mathfrak{a})} * \hat{\psi}_{1}(\mathfrak{a}) \mu_{H}(\mathrm{~d} \mathfrak{a})\right)\left(\alpha_{1}-\alpha_{2}\right)\right\rangle_{L^{2}\left(\Omega_{H} ; \mathbb{C}\right)}
\end{aligned}
$$

with

$$
\psi_{1,2} \doteq U_{H} \varphi_{1,2} \quad \text { and } \quad \hat{\psi}_{1,2} \doteq \hat{U}_{H} \hat{\varphi}_{1,2} \in \ell_{\mathrm{ap}}^{2}\left(\mathbb{T}_{n} ; L^{2}\left(\Omega_{H}\right)\right)
$$

In the r.h.s. of (58) observe that $\hat{\psi}_{1}$ is seen as an element of $L^{2}\left(\Omega_{H} ; \ell_{\text {ap }}^{2}\left(\mathbb{T}_{n} ; \mathbb{C}\right)\right)$, see (53). By (3), observe that

$$
\left[\hat{\psi}_{1}(\mathfrak{a})\right](\alpha)=\delta_{\text {ap }}(\alpha) \cdot\left(U_{H} \varphi_{1}\right)(\mathfrak{a}), \quad \mathfrak{a} \in \Omega_{H}, \alpha \in \mathbb{T}_{n},
$$

which, combined with Equations (5) and (58), yields

$$
\begin{aligned}
& \left\langle\varphi_{2},\left(C_{H} \hat{\varphi}_{1}\right)\left(\alpha_{1}-\alpha_{2}\right)\right\rangle_{\mathfrak{h}} \\
= & \left\langle\psi_{2},\left(\int_{\Omega_{H}}^{\oplus} g_{\lambda_{H}(\mathfrak{a})}\left(\alpha_{1}-\alpha_{2}\right) \psi_{1}(\mathfrak{a}) \mu_{H}(\mathrm{~d} \mathfrak{a})\right)\right\rangle_{L^{2}\left(\Omega_{H} ; \mathbb{C}\right)} .
\end{aligned}
$$

Therefore, by using the explicit expressions (16), (22)-(23) and (56), we deduce from (52) and (59) the equality

$$
\left\langle\varphi_{2},\left(C_{H} \hat{\varphi}_{1}\right)\left(\alpha_{1}-\alpha_{2}\right)\right\rangle_{\mathfrak{h}}=\lim _{\nu \rightarrow \infty}\left\langle\mathrm{E}^{\beta^{-1} n \alpha_{2}+1} \varphi_{2}, \frac{\mathrm{e}^{\left(\alpha_{2}+n^{-1} \beta-\alpha_{1}\right) H_{\nu}}}{1+\mathrm{e}^{\beta H_{\nu}}} \mathrm{E}^{-\beta^{-1} n \alpha_{1}} \varphi_{1}\right\rangle_{\mathfrak{h}}
$$


for any $\alpha_{1} \leq \alpha_{2}$ while, for any $\alpha_{1}>\alpha_{2}$,

$$
\begin{aligned}
& \left\langle\varphi_{2},\left(C_{H} \hat{\varphi}_{1}\right)\left(\alpha_{1}-\alpha_{2}\right)\right\rangle_{\mathfrak{h}} \\
= & -\lim _{\nu \rightarrow \infty}\left\langle\mathrm{E}^{\beta^{-1} n \alpha_{2}+1} \varphi_{2}, \frac{\mathrm{e}^{\beta-\left(\alpha_{1}-\alpha_{2}-n^{-1} \beta\right) H_{\nu}}}{1+\mathrm{e}^{\beta H_{\nu}}} \mathrm{E}^{-\beta^{-1} n \alpha_{1}} \varphi_{1}\right\rangle_{\mathfrak{h}},
\end{aligned}
$$

using $n \in 2 \mathbb{N}$. On the other hand, if $\alpha_{1} \leq \alpha_{2}$ then $\pi_{\alpha_{1}, \alpha_{2}}=\mathbf{1}_{\{1,2\}}$ and we infer from Equations (10), (11), (18), (38) and (39) that

$$
\begin{aligned}
& \rho_{S_{\nu}}\left(\mathbb { O } _ { \pi _ { \alpha _ { 1 } , \alpha _ { 2 } } } \left(a^{+}\left(\left(\mathrm{e}^{-\alpha_{1} H_{\nu}} \mathrm{E}^{-\beta^{-1} n \alpha_{1}} \varphi_{1}\right) \otimes \mathfrak{e}_{j_{1}}\right)\right.\right. \\
\left.\left.a\left(\left(\mathrm{e}^{\left(\alpha_{2}+n^{-1} \beta\right) H_{\nu}} \mathrm{E}^{\beta^{-1} n \alpha_{2}+1} \varphi_{2}\right) \otimes \mathfrak{e}_{j_{2}}\right)\right)\right) & \mathfrak{M}_{j_{1}, j_{2}}\left\langle\mathrm{E}^{\beta^{-1} n \alpha_{2}+1} \varphi_{2}, \frac{\mathrm{e}^{\left(\alpha_{2}+n^{-1} \beta-\alpha_{1}\right) H_{\nu}}}{1+\mathrm{e}^{\beta H_{\nu}}} \mathrm{E}^{-\beta^{-1} n \alpha_{1}} \varphi_{1}\right\rangle_{\mathfrak{h}}
\end{aligned}
$$

and the assertion holds true when $\alpha_{1} \leq \alpha_{2}$. If $\alpha_{1}>\alpha_{2}$ then

$$
\begin{aligned}
\rho_{S_{\nu}}\left(\mathbb { O } _ { \pi _ { \alpha _ { 1 } , \alpha _ { 2 } } } \left(a^{+}\left(\left(\mathrm{e}^{-\alpha_{1} H_{\nu}} \mathrm{E}^{-\beta^{-1} n \alpha_{1}} \varphi_{1}\right) \otimes \mathfrak{e}_{j_{1}}\right),\right.\right. \\
\left.\left.a\left(\left(\mathrm{e}^{\left(\alpha_{2}+n^{-1} \beta\right) H_{\nu}} \mathrm{E}^{\beta^{-1} n \alpha_{2}+1} \varphi_{2}\right) \otimes \mathfrak{e}_{j_{2}}\right)\right)\right) \\
=-\rho_{S_{\nu}}\left(a\left(\left(\mathrm{e}^{\left(\alpha_{2}+n^{-1} \beta\right) H_{\nu}} \mathrm{E}^{\beta^{-1} n \alpha_{2}+1} \varphi_{2}\right) \otimes \mathfrak{e}_{j_{2}}\right)\right. \\
\left.a^{+}\left(\left(\mathrm{e}^{-\alpha_{1} H_{\nu}} \mathrm{E}^{-\beta^{-1} n \alpha_{1}} \varphi_{1}\right) \otimes \mathfrak{e}_{j_{1}}\right)\right),
\end{aligned}
$$

which, combined with (34), implies that

$$
\begin{gathered}
\rho_{S_{\nu}}\left(\mathbb { O } _ { \pi _ { \alpha _ { 1 } , \alpha _ { 2 } } } \left(a^{+}\left(\left(\mathrm{e}^{-\alpha_{1} H_{\nu}} \mathrm{E}^{-\beta^{-1} n \alpha_{1}} \varphi_{1}\right) \otimes \mathfrak{e}_{j_{1}}\right),\right.\right. \\
\left.\left.a\left(\left(\mathrm{e}^{\left(\alpha_{2}+n^{-1} \beta\right) H_{\nu}} \mathrm{E}^{\beta^{-1} n \alpha_{2}+1} \varphi_{2}\right) \otimes \mathfrak{e}_{j_{2}}\right)\right)\right) \\
=\rho_{S_{\nu}}\left(a^{+}\left(\left(\mathrm{e}^{-\alpha_{1} H_{\nu}} \mathrm{E}^{-\beta^{-1} n \alpha_{1}} \varphi_{1}\right) \otimes \mathfrak{e}_{j_{1}}\right)\right. \\
\left.a\left(\left(\mathrm{e}^{\left(\alpha_{2}+n^{-1} \beta\right) H_{\nu}} \mathrm{E}^{\beta^{-1} n \alpha_{2}+1} \varphi_{2}\right) \otimes \mathfrak{e}_{j_{2}}\right)\right) \\
-\left\langle\left(\mathrm{e}^{\left(\alpha_{2}+n^{-1} \beta\right) H_{\nu}} \mathrm{E}^{\beta^{-1} n \alpha_{2}+1} \varphi_{2}\right) \otimes \mathfrak{e}_{j_{2}},\left(\mathrm{e}^{-\alpha_{1} H_{\nu}} \mathrm{E}^{-\beta^{-1} n \alpha_{1}} \varphi_{1}\right) \otimes \mathfrak{e}_{j_{1}}\right\rangle_{\mathfrak{h} \otimes \mathbb{M}} .
\end{gathered}
$$


Using again (10), (11) and (18), we thus arrive from the last equality at

$$
\begin{gathered}
\rho_{S_{\nu}}\left(\mathbb { O } _ { \pi _ { \alpha _ { 1 } , \alpha _ { 2 } } } \left(a^{+}\left(\left(\mathrm{e}^{-\alpha_{1} H_{\nu}} \mathrm{E}^{-\beta^{-1} n \alpha_{1}} \varphi_{1}\right) \otimes \mathfrak{e}_{j_{1}}\right),\right.\right. \\
\left.\left.a\left(\left(\mathrm{e}^{\left(\alpha_{2}+n^{-1} \beta\right) H_{\nu}} \mathrm{E}^{\beta^{-1} n \alpha_{2}+1} \varphi_{2}\right) \otimes \mathfrak{e}_{j_{2}}\right)\right)\right) \\
=-\mathfrak{M}_{j_{1}, j_{2}}\left\langle\mathrm{E}^{\beta^{-1} n \alpha_{2}+1} \varphi_{2}, \frac{\mathrm{e}^{\beta-\left(\alpha_{1}-\alpha_{2}-n^{-1} \beta\right) H_{\nu}}}{1+\mathrm{e}^{\beta H_{\nu}}} \mathrm{E}^{-\beta^{-1} n \alpha_{1}} \varphi_{1}\right\rangle_{\mathfrak{h}} .
\end{gathered}
$$

By combining (60) and (61) with (62) and (63), we arrive at the assertion with $\kappa=\mathbf{1}_{\mathbb{R}}$.

\section{Corollary 2.4 (Determinants of the covariance as quasi-free states)}

Let $\mathfrak{h}$ be any separable Hilbert space. Fix $\beta \in \mathbb{R}^{+}$, a self-adjoint operator $H=H^{*}$ acting on $\mathfrak{h}$, and $n \in 2 \mathbb{N}$. Then, for each bounded measurable positive function $\kappa$ from $\mathbb{R}$ to $\mathbb{R}_{0}^{+}$, all $m, N \in \mathbb{N}$, non-vanishing $\mathfrak{M} \in \operatorname{Mat}(m, \mathbb{R})$ with $\mathfrak{M} \geq 0$ and

$$
\left\{\left(\alpha_{q}, \varphi_{q}, j_{q}\right)\right\}_{q=1}^{2 N} \subset \mathbb{T}_{n} \cap[0, \beta) \times \mathfrak{D} \times\{1, \ldots, m\},
$$

the following identity holds true:

$$
\begin{gathered}
\operatorname{det}\left[\mathfrak{M}_{j_{k}, j_{N+l}}\left\langle\varphi_{N+l},\left(C_{H} \kappa(\hat{H}) \hat{\varphi}_{k}\right)\left(\alpha_{k}-\alpha_{N+l}\right)\right\rangle_{\mathfrak{h}}\right]_{k, l=1}^{N} \\
=\lim _{\nu \rightarrow \infty} \rho_{S_{\nu}}\left(\mathbb { O } _ { \pi } \left(a^{+}\left(\left(\mathrm{e}^{-\tilde{\alpha}_{1} H_{\nu}} \mathrm{E}^{-\beta^{-1} n \tilde{\alpha}_{1}} \varphi_{1}\right) \otimes \mathfrak{e}_{j_{1}}\right), \ldots,\right.\right. \\
a^{+}\left(\left(\mathrm{e}^{-\tilde{\alpha}_{N} H_{\nu}} \mathrm{E}^{-\beta^{-1} n \tilde{\alpha}_{N}} \varphi_{N}\right) \otimes \mathfrak{e}_{j_{N}}\right) \\
a\left(\left(\mathrm{e}^{\tilde{\alpha}_{2 N} H_{\nu}} \mathrm{E}^{\beta^{-1} n \tilde{\alpha}_{2 N}} \varphi_{2 N}\right) \otimes \mathfrak{e}_{j_{2 N}}\right) \\
\left.\left.\ldots, a\left(\left(\mathrm{e}^{\tilde{\alpha}_{N+1} H_{\nu}} \mathrm{E}^{\beta^{-1} n \tilde{\alpha}_{N+1}} \varphi_{N+1}\right) \otimes \mathfrak{e}_{j_{N+1}}\right)\right)\right)
\end{gathered}
$$

for any permutation $\pi$ of $2 N$ elements such that

$$
\tilde{\alpha}_{\pi^{-1}(q)}-\tilde{\alpha}_{\pi^{-1}(q-1)} \geq 0, \quad \alpha_{\pi^{-1}(q)}-\alpha_{\pi^{-1}(q-1)} \geq 0, \quad q \in\{2, \ldots, 2 N\},
$$

where $\tilde{\alpha}_{q} \doteq \alpha_{q}$ for $q \in\{1, \ldots, N\}$ and $\tilde{\alpha}_{q} \doteq \alpha_{q}+n^{-1} \beta$ for $q \in\{N+1, \ldots, 2 N\}$. 
Proof: Fix all the parameters of the corollary. Take any permutation $\pi$ of $2 N$ elements such that

$$
\pi_{\alpha_{k}, \alpha_{N+l}}=\pi_{k, N+l}, \quad k, l \in\{1, \ldots, N\} .
$$

See, respectively, (iv) before Lemma 2.3 and Equation (40) for the definitions of the permutations $\pi_{\alpha_{k}, \alpha_{N+l}}$ and $\pi_{k, N+l}$ of two elements. Then, (64) follows from Lemmata 2.2 and 2.3. To conclude the proof observe that a permutation $\pi$ of $2 N$ elements satisfying (65) exists and also satisfies (66), keeping in mind Equation (1).

\subsection{Correlation Functions and Tomita-Takesaki Modular The- ory}

(i): As above, fix $\beta \in \mathbb{R}^{+}$, a self-adjoint operator $H=H^{*}$ acting on $\mathfrak{h}, n \in 2 \mathbb{N}$, $\overline{\nu \in} \mathbb{R}^{+}$, and a non-vanishing positive real matrix $\mathfrak{M} \in \operatorname{Mat}(m, \mathbb{R})$ with $m \in \mathbb{N}$. Let $\tau \equiv\left\{\tau_{t}\right\}_{t \in \mathbb{R}}$ be the unique $C_{0}$-group (that is, strongly continuous group) of automorphisms on the $C^{*}$-algebra $\operatorname{CAR}(\mathfrak{h} \otimes \mathbb{M})$ satisfying

$$
\tau_{t}(a(\varphi \otimes g))=a\left(\mathrm{e}^{i t H_{\nu} \otimes \mathbf{1}_{\mathfrak{h} \otimes \mathbb{M}}} \varphi \otimes g\right)=a\left(\left(\mathrm{e}^{i t H_{\nu}} \varphi\right) \otimes g\right), \quad \varphi \in \mathfrak{h}, g \in \mathbb{M} .
$$

See (16). It is well-known that the quasi-free state $\rho_{S_{\nu}}$, which is defined from the symbol $S_{\nu}(18)$, is the unique $(\tau, \beta)-\mathrm{KMS}$ state on $\mathrm{CAR}(\mathfrak{h} \otimes \mathbb{M})$.

(ii): Let $\left(\mathfrak{H}_{\nu}, \varkappa_{\nu}, \eta_{\nu}\right)$ be a cyclic representation of $\rho_{S_{\nu}}$. The weak closure of the $C^{*}$-algebra $\mathrm{CAR}(\mathfrak{h} \otimes \mathbb{M})$ is the von Neumann algebra

$$
\mathcal{X}_{\nu}=\varkappa_{\nu}(\mathrm{CAR}(\mathfrak{h} \otimes \mathbb{M}))^{\prime \prime} \subset \mathcal{B}\left(\mathfrak{H}_{\nu}\right) .
$$

The state $\rho_{S_{\nu}} \circ \varkappa_{\nu}$ on $\varkappa_{\nu}(\mathrm{CAR}(\mathfrak{h} \otimes \mathbb{M}))$ extends uniquely to a normal state on the von Neumann algebra $\mathcal{X}_{\nu}$ and the $C_{0}$-group $\left\{\tau_{t} \circ \varkappa_{\nu}\right\}_{t \in \mathbb{R}}$ also uniquely extends to a $\sigma$-weakly continuous $*$-automorphism group on $\mathcal{X}_{\nu}$. Both extensions are again denoted by $\rho_{S_{\nu}}$ and $\left\{\tau_{t}\right\}_{t \in \mathbb{R}}$, respectively. By [BR2, Corollary 5.3.4], $\rho_{S_{\nu}}$ is again a $(\tau, \beta)-$ KMS state on $\mathcal{X}_{\nu}$.

(iii): By [BR2, Corollary 5.3.9], the cyclic vector $\eta_{\nu}$ is separating for $\mathcal{X}_{\nu}$, i.e., $\overline{A \eta_{\nu}}=0$ implies $A=0$ for all $A \in \mathcal{X}_{\nu}$. Denote by $\Delta_{\nu}$ the (possibly unbounded) Tomita-Takesaki modular operator of the pair $\left(\mathcal{X}_{\nu}, \eta_{\nu}\right)$. The $(\beta$-rescaled) modular group is the $\sigma$-weakly continuous one-parameter group $\sigma \equiv\left\{\sigma_{t}\right\}_{t \in \mathbb{R}}$ defined by

$$
\sigma_{t}(A) \doteq \Delta_{\nu}^{-i t \beta^{-1}} A \Delta_{\nu}^{i t \beta^{-1}} \quad A \in \mathcal{X}_{\nu}
$$


(If $\beta=-1$ then $\sigma$ is the well-known modular automorphism group associated with the pair $\left(\mathcal{X}_{\nu}, \eta_{\nu}\right)$, see [BR1, Definition 2.5.15].) By Takesaki's theorem [BR2, Theorem 5.3.10], we deduce that $\sigma=\tau$. In particular, using (67) we arrive at the equality

$$
\varkappa_{\nu}\left(a\left(\left(\mathrm{e}^{i t H_{\nu}} \varphi\right) \otimes g\right)\right)=\Delta_{\nu}^{-i t \beta^{-1}} \varkappa_{\nu}(a(\varphi \otimes g)) \Delta_{\nu}^{i t \beta^{-1}}, \quad \varphi \in \mathfrak{h}, g \in \mathbb{M} .
$$

(iv): Recall that $\mathfrak{D} \subseteq \mathfrak{h}(57)$ is a dense subspace of entire analytic vectors for $H_{\nu}$, while for any $N \in \mathbb{N}$ and $\zeta \in \mathbb{R}^{+}, \mathfrak{T}_{N}^{(\zeta)}$ is the tube defined by (31). For any $\varphi \in \mathfrak{D}$ and $g \in \mathbb{M}$, the maps

$$
z \mapsto a^{+}\left(\left(\mathrm{e}^{-z H_{\nu}} \varphi\right) \otimes g\right) \quad \text { and } \quad z \mapsto a\left(\left(\mathrm{e}^{\bar{z} H_{\nu}} \varphi\right) \otimes g\right)
$$

from $\mathbb{C}$ to the $C^{*}$-algebra $\operatorname{CAR}(\mathfrak{h} \otimes \mathbb{M})$ are entire analytic functions. Fix $N \in \mathbb{N}$, $\varphi_{1}, \ldots, \varphi_{N} \in \mathfrak{D}$ and $g_{1}, \ldots, g_{N} \in \mathbb{M}$ and pick a family

$$
\left\{a^{\#}\left(\varphi_{q} \otimes g_{q}\right)\right\}_{q=1}^{N} \subset \mathrm{CAR}(\mathfrak{h} \otimes \mathbb{M}),
$$

where the notation " $a$ " " stands for either " $a$ " " or " $a$ ". For any $\varphi \in \mathfrak{D}, g \in \mathbb{M}$ and $z \in \mathbb{C}$, we also use the convention

$$
a^{\#}\left(\left(\mathrm{e}^{z^{\#} H_{\nu}} \varphi\right) \otimes g\right)=\left\{\begin{array}{lll}
a^{+}\left(\left(\mathrm{e}^{-z H_{\nu}} \varphi\right) \otimes g\right) & \text { when } a^{\#}=a^{+}, \\
a\left(\left(\mathrm{e}^{\bar{z} H_{\nu}} \varphi\right) \otimes g\right) & \text { when } a^{\#}=a,
\end{array}\right.
$$

with

$$
\left(z_{1}+z_{2}\right)^{\#} \doteq z_{1}^{\#}+z_{2}^{\#}, \quad z_{1}, z_{2} \in \mathbb{C} .
$$

Then, for any fixed integer $p \in\{1, \ldots, N\}$, the map $\Upsilon$ from $\mathbb{C}^{N+1}$ to $\mathbb{C}$ defined by

$$
\begin{aligned}
& \Upsilon\left(z_{1}, \ldots, z_{p}, \tilde{z}_{p}, z_{p+1}, \ldots, z_{N}\right) \\
& \doteq \rho\left(a^{\#}\left(\left(\mathrm{e}^{z_{1}^{\#} H_{\nu}} \varphi_{1}\right) \otimes g_{1}\right) \cdots a^{\#}\right.\left(\left(\mathrm{e}^{\left(z_{1}^{\#}+\cdots+z_{p-1}^{\#}\right) H_{\nu}} \varphi_{p-1}\right) \otimes g_{p-1}\right) \\
& a^{\#}\left(\left(\mathrm{e}^{\left(z_{1}^{\#}+\cdots+z_{p}^{\#}+z_{p}^{\#}\right) H_{\nu}} \varphi_{p}\right) \otimes g_{p}\right) a^{\#}\left(\left(\mathrm{e}^{\left(z_{1}^{\#}+\cdots+z_{p}^{\#}+z_{p}^{\#}+z_{p+1}^{\#}\right) H_{\nu}} \varphi_{p+1}\right) \otimes g_{p+1}\right) \\
&\left.\cdots a^{\#}\left(\left(\mathrm{e}^{\left(z_{1}^{\#}+\cdots+z_{p}^{\#}+z_{p}^{\#}+z_{p+1}^{\#}+\cdots+z_{N}^{\#}\right) H_{\nu}} \varphi_{N}\right) \otimes g_{N}\right)\right)
\end{aligned}
$$

is an entire analytic function.

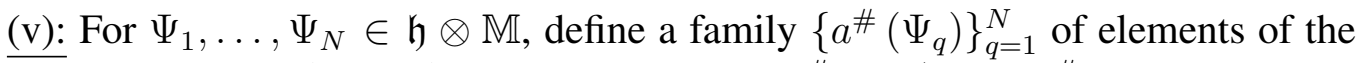
$\overline{C^{*}}$-algebra $\operatorname{CAR}(\mathfrak{h} \otimes \mathbb{M})$, where, as before, " $a^{\#}=a^{+}$" or " $a$ \# $=a$ ". Then, by applying [AM, Lemma A.1], we obtain the following assertions: 
(A) The (cyclic and separating) vector $\eta_{\nu}$ belongs to the domain of definition of the possibly unbounded operator

$$
\begin{aligned}
& \qquad \Delta_{\nu}^{z_{1} \beta^{-1}} \varkappa_{\nu}\left(a^{\#}\left(\Psi_{1}\right)\right) \cdots \Delta_{\nu}^{z_{N} \beta^{-1}} \varkappa_{\nu}\left(a^{\#}\left(\Psi_{N}\right)\right) \\
& \text { for any }\left(z_{1}, \ldots, z_{N}\right) \in \mathfrak{T}_{N}^{(\beta / 2)} \text { with } \\
& \left\|\Delta_{\nu}^{z_{1} \beta^{-1}} \varkappa_{\nu}\left(a^{\#}\left(\Psi_{1}\right)\right) \cdots \Delta_{\nu}^{z_{N} \beta^{-1}} \varkappa_{\nu}\left(a^{\#}\left(\Psi_{N}\right)\right) \eta_{\nu}\right\|_{\mathfrak{H}_{\nu}} \leq \prod_{q=1}^{N}\left\|\Psi_{q}\right\|_{\mathfrak{h} \otimes \mathbb{M}} .
\end{aligned}
$$

This inequality is a special case of [AM, (A.2)], which is intimately related to Hölder inequalities for non-commutative $L^{p}$-spaces.

(B) The map from $\mathfrak{T}_{N}^{(\beta / 2)}$ to $\mathfrak{H}_{\nu}$ defined by

$$
\left(z_{1}, \ldots, z_{N}\right) \mapsto \Delta_{\nu}^{z_{1} \beta^{-1}} \varkappa_{\nu}\left(a^{\#}\left(\Psi_{1}\right)\right) \cdots \Delta_{\nu}^{z_{N} \beta^{-1}} \varkappa_{\nu}\left(a^{\#}\left(\Psi_{N}\right)\right) \eta_{\nu}
$$

is norm continuous on the whole tube $\mathfrak{T}_{N}^{(\beta / 2)}$ and analytic on its interior.

Using the notation

$$
x_{q} \doteq \varkappa_{\nu}\left(a^{\#}\left(\varphi_{q} \otimes g_{q}\right)\right), \quad q \in\{1, \ldots, N\}
$$

we consider now the map $\Theta$ from $\mathfrak{T}_{p}^{(\beta / 2)} \times \mathfrak{T}_{N-p+1}^{(\beta / 2)}$ to $\mathbb{R}$ defined by

$$
\begin{aligned}
& \Theta\left(\left(z_{1}, \ldots, z_{p}\right),\right.\left.\left(\tilde{z}_{p}, z_{p+1}, \ldots, z_{N}\right)\right) \\
& \doteq\left\langle\Delta_{\nu}^{\bar{z}_{p} \beta^{-1}} x_{p-1}^{*} \Delta_{\nu}^{\bar{z}_{p-1} \beta^{-1}} \cdots x_{2}^{*} \Delta_{\nu}^{\bar{z}_{2} \beta^{-1}} x_{1}^{*} \eta_{\nu}\right. \\
&\left.\Delta_{\nu}^{\tilde{z}_{p} \beta^{-1}} x_{p} \Delta_{\nu}^{z_{p+1} \beta^{-1}} x_{p+1} \cdots \Delta_{\nu}^{z_{N} \beta^{-1}} x_{N} \eta_{\nu}\right\rangle_{\mathfrak{H}_{\nu}}
\end{aligned}
$$

Compare for instance with [AM, Lemma A], which explains the properties of $\Theta$. (Notice that $[\mathrm{AM}]$ uses a different convention for sesquilinear forms.) By (73), this function is uniformly bounded for all $n \in 2 \mathbb{N}$. The same is trivially true for the map $\Upsilon$ (72) on

$$
\mathfrak{T}_{p}^{(\beta / 2)} \times \mathfrak{T}_{N-p+1}^{(\beta / 2)} \subset \mathbb{C}^{N+1} .
$$

Moreover, by using (69) we deduce that $\Upsilon$ and $\Theta$ are equal to each other on $i \mathbb{R}^{p} \times i \mathbb{R}^{N-p+1}$. For each fixed imaginary vector $\left(z_{1}, \ldots, z_{p}\right) \in i \mathbb{R}^{p}$, the maps 
$\Upsilon$ and $\Theta$ are both continuous as functions of $\left(\tilde{z}_{p}, z_{p+1}, \ldots, z_{N}\right) \in \mathfrak{T}_{N-p+1}^{(\beta / 2)}$ and analytic in the interior of $\mathfrak{T}_{N-p+1}^{(\beta / 2)}$, by (B) [AM, Lemma A.1]. Hence, from Hadamard's three line theorem (see, e.g., [RS2, Appendix to IX.4]), $\Upsilon$ and $\Theta$ are equal to each other for any fixed imaginary vector $\left(z_{1}, \ldots, z_{p}\right) \in i \mathbb{R}^{p}$ and all complex vectors $\left(\tilde{z}_{p}, z_{p+1}, \ldots, z_{N}\right) \in \mathfrak{T}_{N-p+1}^{(\beta / 2)}$. Applying this argument again at fixed $\left(\tilde{z}_{p}, z_{p+1}, \ldots, z_{N}\right) \in \mathfrak{T}_{N-p+1}^{(\beta / 2)}$ for $\Upsilon$ and $\Theta$ viewed as functions of $\left(z_{1}, \ldots, z_{p}\right) \in$ $\mathfrak{T}_{p}^{(\beta / 2)}$, we conclude that $\Upsilon=\Theta$ on $\mathfrak{T}_{p}^{(\beta / 2)} \times \mathfrak{T}_{N-p+1}^{(\beta / 2)}$.

In particular, for $N \in \mathbb{N}$, any family (71) of elements of the $C^{*}$-algebra $\operatorname{CAR}(\mathfrak{h} \otimes \mathbb{M})$, and all $\alpha_{1}, \ldots, \alpha_{N} \in[0, \beta]$ such that

$$
\vartheta_{q} \doteq \beta^{-1}\left(\alpha_{q}-\alpha_{q-1}\right) \geq 0, \quad q \in\{2, \ldots, N\},
$$

the following equality holds true:

$$
\begin{gathered}
\rho\left(a^{\#}\left(\left(\mathrm{e}^{\alpha_{1}^{\#} H_{\nu}} \varphi_{1}\right) \otimes g_{1}\right) \ldots a^{\#}\left(\left(\mathrm{e}^{\alpha_{N}^{\#} H_{\nu}} \varphi_{N}\right) \otimes g_{N}\right)\right) \\
=\left\langle\Delta_{\nu}^{\frac{1}{2}-\beta^{-1} \alpha_{p-1}} x_{p-1}^{*} \Delta_{\nu}^{\vartheta_{p-1}} \cdots x_{2}^{*} \Delta_{\nu}^{\vartheta_{2}} x_{1}^{*} \eta_{\nu}\right. \\
\left.\Delta_{\nu}^{\beta^{-1} \alpha_{p}-\frac{1}{2}} x_{p} \Delta_{\nu}^{\vartheta_{p+1}} x_{p+1} \cdots \Delta_{\nu}^{\vartheta_{N}} x_{N} \eta_{\nu}\right\rangle_{\mathfrak{H}_{\nu}}
\end{gathered}
$$

with $p$ defined to be the smallest element of $\{1, \ldots, N\}$ such that $\alpha_{p} \geq \beta / 2$.

Acknowledgments: This research is supported by the FAPESP, the Basque Government through the grant IT641-13 and the BERC 2014-2017 program and by the Spanish Ministry of Economy and Competitiveness MINECO: BCAM Severo Ochoa accreditation SEV-2013-0323 and MTM2014-53850. We are very grateful to the BCAM and its management, which supported this project via the visiting researcher program. Finally, we thank Zosza Lefevre for linguistic hints and Christian Jäkel for the nice lectures he gave in 2015 on non-commutative $L^{p_{-}}$ spaces.

\section{References}

[AR] A. Abdesselam and V. Rivasseau, Trees, forests and jungles: a botanical garden for cluster expansions. Constructive physics (Palaiseau, 1994), 7-36, Lecture Notes in Phys., 446, Springer, Berlin, 1995.

[A] H. ARAKI, On Quasifree States of CAR and Bogoliubov Automorphisms, Publ. RIMS, Kyoto Univ., 6 (1970/71), 385-442. 
[AM] H. Araki And T. Masuda, Positive Cones and $L_{p}$-Spaces for von Neumann Algebras, Publ. RIMS, Kyoto Univ., 18 (1982), 339-411.

[BGPS] G. Benfatto, G. Gallavotti, A. Procacci, and B. Scoppola, Beta function and Schwinger functions for a many fermions system in one dimension. Anomaly of the Fermi surface, Comm. Math. Phys., 160(1) (1994) 93-171.

[BR1] O. BRATTELI AND D.W. Robinson, Operator Algebras and Quantum Statistical Mechanics, Vol. I, 2nd ed. Springer-Verlag, New York, 1996.

[BR2] O. BRATteli And D.W. Robinson, Operator Algebras and Quantum Statistical Mechanics, Vol. II, 2nd ed. Springer-Verlag, New York, 1996.

[BK] D. C. BRydges And T. Kennedy, Mayer Expansions and the Hamilton-Jacobi Equation, J. Stat. Phys., 48 (1/2) (1987) 19-49.

[DF] J. DEREZINSKI AND R. Früboes, Fermi Golden Rule and Open Quantum Systems. In Open Quantum Systems III, Volume 1882, pages 67116. Springer-Verlag, 2006.

[dSP] W. DE SiqueIRA PEdRA, Zur mathematischen Theorie der Fermiflüssigkeiten bei positiven Temperaturen. PhD Thesis, University of Leipzig, 2005.

[dSPS] W. De Siqueira Pedra And M. Salmhofer, Determinant Bounds and the Matsubara UV Problem of Many-Fermion Systems, Commun. Math. Phys. 282 (2008) 797-818.

[GM] A. Giuliani And V. Mastropietro, The Two-Dimensional Hubbard Model on the Honeycomb Lattice, Commun. Math. Phys. 293 (2010) 301-346.

[GMP] A. Giuliani, V. Mastropietro and M. Porta, Universality of the Hall conductivity in interacting electron systems, arXiv: $1511.04047 \mathrm{v} 2$ [math-ph] 20 Dec 2015.

[RS2] M. REed AND B. SIMON, Methods of Modern Mathematical Physics, Vol. II: Fourier Analysis, Self-Adjointness. Academic Press, New YorkLondon, 1975. 
[S] M. SAlmhofer, Renormalization, An Introduction, Springer-Verlag Berlin Heidelberg, 1999.

[SW] M. Salmhofer And C. Wieczerkowski, Positivity and Convergence in Fermionic Quantum Field Theory, J. Stat. Phys., 99(1/2) (2000) $557-586$. 